OPEN ACCESS

Edited by:

David Lutz,

Ruhr-University Bochum, Germany

Reviewed by:

Jorge Valero,

Achucarro Basque Center

for Neuroscience, Spain

Tzong-Shive Yu,

Columbia University, United States

*Correspondence:

Kathryn E. Saatman

k.saatman@uky.edu

Specialty section:

This article was submitted to

Signaling,

a section of the journal

Frontiers in Cell and Developmental

Biology

Received: 02 February 2021

Accepted: 26 April 2021

Published: 20 May 2021

Citation:

Littlejohn EL, DeSana AJ,

Williams HC, Chapman RT, Joseph B,

Juras JA and Saatman KE (2021)

IGF1-Stimulated Posttraumatic

Hippocampal Remodeling Is Not

Dependent on MTOR.

Front. Cell Dev. Biol. 9:663456.

doi: 10.3389/fcell.2021.663456

\section{IGF1-Stimulated Posttraumatic Hippocampal Remodeling Is Not Dependent on mTOR}

\author{
Erica L. Littlejohn, Anthony J. DeSana, Hannah C. Williams, Rudy T. Chapman, \\ Binoy Joseph, Jelena A. Juras and Kathryn E. Saatman*
}

Department of Physiology, Spinal Cord and Brain Injury Research Center, University of Kentucky, Lexington, $K Y$, United States

Adult hippocampal neurogenesis is stimulated acutely following traumatic brain injury (TBI). However, many hippocampal neurons born after injury develop abnormally and the number that survive long-term is debated. In experimental TBI, insulin-like growth factor1 (IGF1) promotes hippocampal neuronal differentiation, improves immature neuron dendritic arbor morphology, increases long-term survival of neurons born after TBI, and improves cognitive function. One potential downstream mediator of the neurogenic effects of IGF1 is mammalian target of rapamycin (mTOR), which regulates proliferation as well as axonal and dendritic growth in the CNS. Excessive mTOR activation is posited to contribute to aberrant plasticity related to posttraumatic epilepsy, spurring preclinical studies of mTOR inhibitors as therapeutics for TBI. The degree to which pro-neurogenic effects of IGF1 depend upon upregulation of mTOR activity is currently unknown. Using immunostaining for phosphorylated ribosomal protein S6, a commonly used surrogate for mTOR activation, we show that controlled cortical impact TBI triggers mTOR activation in the dentate gyrus in a time-, region-, and injury severity-dependent manner. Posttraumatic mTOR activation in the granule cell layer (GCL) and dentate hilus was amplified in mice with conditional overexpression of IGF1. In contrast, delayed astrocytic activation of mTOR signaling within the dentate gyrus molecular layer, closely associated with proliferation, was not affected by IGF1 overexpression. To determine whether mTOR activation is necessary for IGF1-mediated stimulation of posttraumatic hippocampal neurogenesis, wildtype and IGF1 transgenic mice received the mTOR inhibitor rapamycin daily beginning at 3 days after $\mathrm{TBI}$, following pulse labeling with bromodeoxyuridine. Compared to wildtype mice, IGF1 overexpressing mice exhibited increased posttraumatic neurogenesis, with a higher density of posttrauma-born GCL neurons at 10 days after injury. Inhibition of mTOR did not abrogate IGF1-stimulated enhancement of posttraumatic neurogenesis. Rather, rapamycin treatment in IGF1 transgenic mice, but not in WT mice, increased numbers of cells labeled with BrdU at 3 days after injury that survived to 10 days, and enhanced the proportion of posttrauma-born cells that differentiated into neurons. Because beneficial effects of IGF1 on hippocampal neurogenesis were maintained or even enhanced with delayed inhibition of mTOR, combination therapy approaches may hold promise for TBI.

Keywords: traumatic brain injury, insulin-like growth factor-1, mTOR, neurogenesis, rapamycin, neuronal differentiation, pS6, dendritic outgrowth 


\section{INTRODUCTION}

Annually, millions of people are living with long-term disability caused by traumatic brain injuries (TBI) (Taylor et al., 2017). The disruption of neural networks resulting from axonal and dendritic degeneration, neuronal cell death and a limited ability to replace and repair lost cells contributes to cognitive disability. The brain can respond to injury by stimulating forms of adaptive plasticity such as cellular reorganization, neural cell proliferation, angiogenesis, altered neurotransmitter release, and neurogenesis (Bramlett and Dietrich, 2015; McGinn and Povlishock, 2015). In particular, hippocampal neurogenesis may play an important role in cognitive recovery following trauma. Ablating neurons born after TBI impairs cognitive recovery (Blaiss et al., 2011). This is consistent with work directly linking enhanced neurogenesis to improvements in spatial learning, reference memory and pattern separation (Sahay et al., 2011; Burghardt et al., 2012; Bekinschtein et al., 2014). However, enhanced neurogenesis has also been associated with histological hallmarks of epilepsy (Parent et al., 1997; Jessberger et al., 2005; Butler et al., 2015), raising concern about therapies designed to drive increased hippocampal neurogenesis after brain injury. A better understanding of mechanisms underlying posttraumatic neurogenesis is essential to ensure optimal therapeutic targeting.

Functionally and developmentally appropriate neurogenesis is a complex process, requiring successful completion of various stages, including neural progenitor cell (NPC) proliferation, neuronal differentiation, migration, and integration (Kempermann et al., 2015). Following experimental TBI in adult rodents, cellular proliferation is upregulated in the subgranular zone (SGZ) of the hippocampal dentate gyrus (DG), resulting in increased numbers of posttraumaborn granule neurons (Kernie et al., 2001; Rola et al., 2006). However, within the altered environment of the traumatized brain, many new hippocampal neurons develop with abnormal dendritic arbors, a phenotype maintained into maturity (Carlson et al., 2014; Villasana et al., 2015; Ibrahim et al., 2016). Trauma also influences migration of immature neurons within the granule cell layer (GCL) and promotes ectopic migration of a small subset of new neurons to the hilar layer of the dentate (HL) (Ibrahim et al., 2016; Robinson et al., 2016; Shapiro, 2017; Littlejohn et al., 2020), suggesting that interventions to improve the fidelity of neurogenic responses after TBI may have utility in guiding appropriate neuronal positioning.

Insulin-like growth factor-1 (IGF1) is an endogenous growth factor that promotes neural plasticity. IGF1 stimulates neurogenesis, process outgrowth, and immature neuron migration in the developing subventricular zone (SVZ) and in hippocampal cell cultures (Hurtado-Chong et al., 2009; Nieto-Estevez et al., 2016). To investigate the efficacy of IGF1 to promote posttraumatic neurogenesis, we have utilized a transgenic mouse with a tet-off construct in which expression of human IGF1 is driven by the GFAP promoter upon the removal of doxycycline-supplemented chow, resulting in postnatal conditional overexpression of IGF1 by astrocytes
(Ye et al., 2004). In the controlled cortical impact (CCI) model of contusion TBI, injury triggers a robust wave of reactive astrocytosis in the contused cortex and underlying hippocampus (Dunn-Meynell and Levin, 1997; Myer et al., 2006; Saatman et al., 2006). In this transgenic mouse model, reactive astrocytes upregulate GFAP, thereby driving regional IGF1 overexpression targeted to damaged tissue regions. We have shown a progressive elevation of hippocampal IGF1 levels in astrocyte-specific IGF1 transgenic (IGFtg) mice over the first 3 days after CCI (Madathil et al., 2013). We have further shown that brain-specific, astrocyte-driven IGF1 overexpression stimulates neuronal differentiation, enhances immature neuron dendritic development and improves long-term survival of hippocampal neurons born after contusion TBI (Carlson et al., 2014; Littlejohn et al., 2020). The neurogenic effects of IGF1 were confirmed using a continuous intracerebroventricular infusion of IGF1 to mice after CCI brain injury, resulting in significantly improved recovery of the immature neuron population within the DG (Carlson and Saatman, 2018). Although IGF1 overexpression potentiates injury-induced Akt activity in the hippocampus (Madathil et al., 2013), little is known regarding molecular pathways underlying neurogenic effects of IGF1 in TBI.

The mammalian target of rapamycin (mTOR), a signaling molecule downstream of Akt, is a potent modulator of cell homeostasis and regulates cell growth, energy expenditure, and survival (Lipton and Sahin, 2014). In vitro studies have defined a role for mTOR signaling in the regulation of neuronal differentiation, migration, dendritic outgrowth, and survival of newly born neurons (Jaworski et al., 2005; Wahane et al., 2014). mTOR activity is transiently increased within the first day(s) after TBI (Chen et al., 2007; Park et al., 2012; Zhu et al., 2014; Nikolaeva et al., 2016; Wang et al., 2016) and is required for early proliferation of progenitor cells in the SGZ (Wang et al., 2016). However, mTOR activation is postulated to contribute to posttraumatic maladaptive hippocampal plasticity and cognitive impairment associated with posttraumatic epilepsy, a premise supported by improved outcomes associated with administration of the mTOR inhibitor rapamycin (Park et al., 2012; Guo et al., 2013; Butler et al., 2015; Rozas et al., 2015). In light of the potential role for mTOR activity in stimulating aberrant axonal sprouting of granule cell neurons, an outcome that could increase the likelihood of posttraumatic epilepsy, a therapy that drives mTOR activity could be contraindicated for TBI.

To determine whether IGF1 mediates its effects on posttraumatic neurogenesis through mTOR signaling, we subjected mice with astrocyte-specific, conditional IGF1 overexpression to CCI brain injury. We first mapped the patterns, time course, and dependence on injury severity of mTOR activation in the DG, using immunolabeling for phosphorylation of a downstream target, ribosomal protein S6. We then inhibited posttraumatic mTOR activation using a delayed administration of rapamycin, and queried the effects on early hippocampal neurogenesis in wildtype and IGF1 transgenic mice. 


\section{MATERIALS AND METHODS}

\section{Animals}

Heterozygous tTA ${ }^{\text {GFAP }}$ mice were bred with heterozygous $\mathrm{IGF}^{\mathrm{pTRE}}$ mice as previously described (Ye et al., 2004; Madathil et al., 2013) to generate Tet-off double transgenic

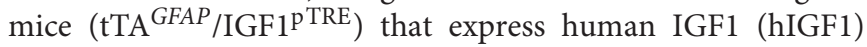
selectively in astrocytes. IGF1 double transgenic (IGFtg) mice and their wildtype (WT) littermates were fed with doxycycline supplemented mouse chow $(200 \mathrm{mg} / \mathrm{kg})$ ad libitum until 2 weeks prior to surgery to block hIGF1 transcription. They received standard mouse chow for at least 2 weeks to allow transgene expression prior to surgery/injury. In the absence of doxycycline, hIGF is expressed, but at low levels in uninjured mice due to low basal levels of glial fibrillary acidic protein (GFAP) transcription. In contrast, in mice with CCI, reactive astrocytosis triggers upregulation of GFAP production, resulting in concomitant increases in IGF1 levels in injured regions such as the hippocampus (Madathil et al., 2013).

Mice were provided with food and water ad libitum at the University of Kentucky Medical Center animal vivarium where they were housed at a constant temperature $\left(23 \pm 2^{\circ} \mathrm{C}\right)$ with a 14/10-h light/dark cycle. All procedures involving animals were approved by the University of Kentucky's Institutional Animal Care and Use Committee, under IACUC protocols 20131156 and 2019-3293.

\section{Controlled Cortical Impact Injury}

Adult ( $>8$ weeks of age) male and female littermates were randomly assigned to CCI or sham injury groups. Surgeons blinded to mouse genotypes and treatments performed moderate or severe CCI on IGFtg $(n=59)$ and WT $(n=57)$ mice. Surgeries were performed as previously described (Madathil et al., 2013). Briefly, anesthesia was induced using $3 \%$ isoflurane. After securing the head of the animal in a stereotaxic frame (David Kopf Instruments, CA), anesthesia was maintained using 2.5\% isoflurane delivered through a nose cone. A midline scalp incision was made, and a $5 \mathrm{~mm}$ diameter craniotomy was performed over the left parietal cortex, lateral to the sagittal suture $(2.5 \mathrm{~mm}$ lateral) midway between Bregma and Lambda. Cortical contusion was produced using a stereotaxic electromagnetic impactor (Leica Biosystems) with a $3 \mathrm{~mm}$ diameter rounded impactor tip, with a velocity of $3.5 \mathrm{~m} / \mathrm{s}$ to produce moderate (1.0$1.1 \mathrm{~mm}$ depth) or severe (1.5 mm depth) brain injury (Figure 1). IGFtg $(n=5)$ and WT $(n=7)$ sham-injured mice received a craniotomy under isoflurane anesthesia. After CCI or sham injury, a circular disk made from dental cement was glued over the craniotomy to protect the brain surface, and the scalp was sutured. Mice were placed on a Hova-Bator Incubator (model 1583, Randall Burkey Co., TX) to maintain body temperature until they regained consciousness, after which they were returned to their home cages. Sham mice for the pS6 immunostaining experiment (Figure 1A) were euthanized 3 days after surgery.

\section{Rapamycin Administration}

Rapamycin (LC Laboratories, Woburn, MA) was dissolved in $100 \%$ ethanol $(30 \mathrm{mg} / \mathrm{ml})$ and stored at $-20^{\circ} \mathrm{C}$ until use.
Immediately before injection, rapamycin was diluted (1:10) into vehicle (5\% Tween $80,5 \%$ PEG400, 5\% EtOH in $1 \times$ PBS). Moderately injured IGFtg and WT mice in a 3-day (Figure 1B) and a 10-day (Figure 1C) survival cohort were randomly assigned to either vehicle or rapamycin treatment groups for each experiment. The 3-day survival cohort received daily intraperitoneal (i.p.) injections of $10 \mathrm{mg} / \mathrm{kg}$ rapamycin ( $n=3$ /genotype $)$ or vehicle $(n=3$ /genotype $)$ beginning $1 \mathrm{~h}$ after CCI. The 10-day survival cohort mice received a daily i.p. injection of $10 \mathrm{mg} / \mathrm{kg}$ rapamycin ( $n=8 /$ genotype) or vehicle ( $n=6-7 /$ genotype) beginning $1 \mathrm{~h}$ after the last BrdU injection.

\section{BrdU Administration}

The 10-day survival cohort received three i.p. injections of $50 \mathrm{mg} / \mathrm{kg}$ 5-Bromo-2'-deoxyuridine (BrdU, Fisher Scientific, Hampton $\mathrm{NH}$ ) in saline at $4 \mathrm{~h}$ intervals on day 3 after injury (Figure 1C). Intraperitoneal BrdU typically incorporates into dividing cells within $4 \mathrm{~h}$ after injection (Taupin, 2007). During handling and disposal of BrdU and all hazardous materials used in this study, proper precautions were taken as approved by the University of Kentucky Office of Environmental Health and Safety and the Institutional Animal Care and Use Committee.

\section{Immunohistochemistry and Histology}

Animals were deeply anesthetized by sodium pentobarbital (Fatal-plus solution, Vortech Pharmaceuticals, Dearborn, MI) and transcardially perfused with heparinized saline followed by $10 \%$ buffered formalin. Brains were removed $24 \mathrm{~h}$ after postfixation in $10 \%$ formalin, then cryoprotected using $30 \%$ sucrose solution and snap frozen in cold isopentanes $\left(\leq-25^{\circ} \mathrm{C}\right)$. The tissue was cut in a coronal plane at $40 \mu \mathrm{m}$ thickness. Free-floating immunohistochemistry was performed as previously described on three tissue sections (between -1.5 and $-2.9 \mathrm{~mm}$ Bregma) selected at $400 \mu \mathrm{m}$ intervals spanning the injury epicenter (Madathil et al., 2013; Carlson et al., 2014). To increase specific staining of phosphorylated ribosomal protein S6 (pS6), antigen retrieval was performed using $10 \mathrm{mM}$ citric acid $\mathrm{pH} 6.0$ at $60^{\circ} \mathrm{C}$. To expose BrdU epitopes, tissue was incubated in $2 \mathrm{~N} \mathrm{HCl}$ (Fisher Scientific) at room temperature with agitation for $1 \mathrm{~h}$, followed by $100 \mathrm{mM}$ borate for $10 \mathrm{~min}$ to neutralize residual $\mathrm{HCl}$. The tissue was rinsed overnight in TBS at $4^{\circ} \mathrm{C}$. Following pretreatment, sections were washed three times in TBS and then incubated in blocking solution (5\% normal horse serum in $0.1 \%$ Triton X-100 in TBS) for $1 \mathrm{~h}$. The tissue was incubated with primary antibody at $4^{\circ} \mathrm{C}$ overnight. Primary antibodies include: pS6 (1:100 rabbit polyclonal anti-pS6, Cat. \#2215, Cell Signaling, Boston, MA), a well characterized downstream mTOR effector (Chen et al., 2007); BrdU (1:1000 rat monoclonal anti-BrdU, Cat. \#ab6326, Abcam, Boston, MA), a proliferation reporter (Dash et al., 2001); the microtubule protein doublecortin (1:2500 rabbit polyclonal anti-Dcx, Cat. \#ab18723, Abcam), a commonly used immature neuron marker (Kempermann et al., 2015); proliferating cell nuclear antigen (1:500 mouse monoclonal antiPCNA, Cat. \#2586, Cell Signaling), a proliferation marker; NeuN (1:500 mouse monoclonal anti-NeuN, Cat. \#MAB377, EMD Millipore Corporation, Burlington, MA), a marker for mature 
A

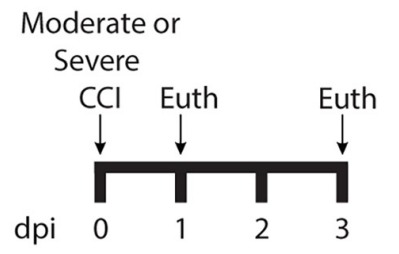

$\mathrm{n}=9-11 \mathrm{CCl} /$ genotype/severity $n=5-7$ Sham/genotype/severity
B

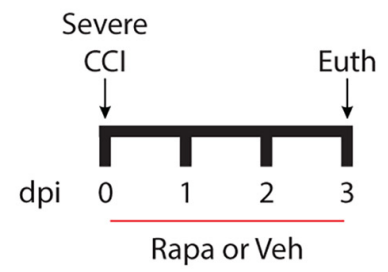

$\mathrm{n}=3 \mathrm{CCl} /$ genotype/treatment

C

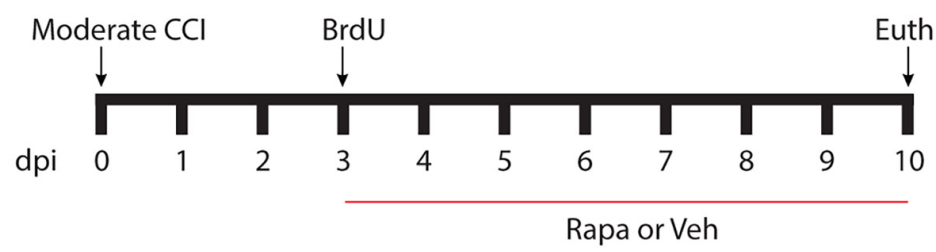

$\mathrm{n}=6-8 \mathrm{CCl} /$ genotype/treatment

D
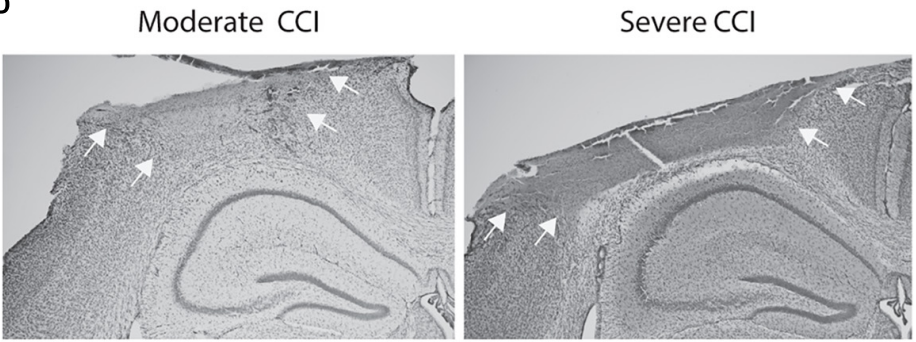

FIGURE 1 | Schematic of experimental design. (A) To assess acute posttraumatic mTOR activity, cohorts of mice were euthanized 24 or $72 \mathrm{~h}$ after moderate or severe controlled cortical impact (CCl) injury. (B) To verify mTOR inhibition by rapamycin (Rapa), brain-injured mice were treated daily with $10 \mathrm{mg} / \mathrm{kg}$ Rapa or vehicle (Veh) beginning $1 \mathrm{~h}$ after injury for 3 days. (C) To examine the contribution of mTOR signaling to posttraumatic neurogenesis, injured mice were treated daily with $10 \mathrm{mg} / \mathrm{kg}$ Rapa or Veh beginning on day 3, after mice received three injections of $50 \mathrm{mg} / \mathrm{kg}$ BrdU given $4 \mathrm{~h}$ apart. dpi, days post-injury. (D) Representative images taken from mice with moderate (left) and severe (right) CCl injury. Nissl staining reveals a well-developed cortical contusion at the site of impact, which is larger following severe $\mathrm{CCl}$ as compared to moderate $\mathrm{CCl}$. Arrows illustrate the boundaries of the contusion.

neurons; Iba1 (1:1,000 rabbit polyclonal anti-Iba1, Cat. \#01919741, FUJIFILM Wako Pure Chemical Corporation, Richmond, VA), a microglia marker; and glial fibrillary acidic protein (1:1,000 rabbit polyclonal anti-GFAP, Cat. \#G9269, EMD Millipore or 1:1,000 chicken polyclonal anti-GFAP, Cat. \#GFAP, Aves Labs, Inc., Davis, CA), an astrocyte marker. Secondary antibodies were conjugated with Alexa-488, Cy-3, or Alexa-594 (Invitrogen, Carlsbad, CA). Omission of primary antibody served as a negative control.

\section{Microscopy Image Analysis}

All image acquisition and subsequent analyses were completed by an investigator blinded to the genotype and treatment status of each animal.

\section{Positive Immunoreactive Area Imaging and Analysis}

For all pS6 analyses, image acquisition was performed using NIS-Elements software (Nikon Instruments, Inc., Melville, NY).
After identifying the center of the section, profile images of the DG were taken at $20 \times$ magnification (NA 0.75, Nikon, Melville, $\mathrm{NY}$ ) using a laser scanning confocal microscope equipped with a photomultiplier tube (Nikon Ti Eclipse with C2 plus). All images were captured using identical laser and photodetector settings. Ipsilateral and contralateral hemispheres in each section were imaged, and on a composite image several regions of interest (ROI) were delineated: granule cell layer (GCL), molecular layer (ML), and hilus (HL). Within each ROI, a threshold was set to capture pS6 + cells and a binary mask was applied (see Supplementary Figure 1$)$. The area $\left(\mathrm{mm}^{2}\right)$ of positive pixels was quantified and normalized to the ROI area $\left(\mathrm{mm}^{2}\right)$. Data was averaged from 3 sections per animal.

\section{Immature Neuron Image Acquisition and Cell Counts}

For all BrdU +, Dcx +, and BrdU + Dcx + cell analyses, the entire ipsilateral DG of the three sections representing the epicenter of injury (Carlson et al., 2014) was imaged on a 
confocal microscope (Nikon A1R). Images were acquired as a $\mathrm{z}$-stack at $100 \times$ magnification under oil (NA 1.45) and at $0.75 \mu \mathrm{m}$ step intervals through a $20 \mu \mathrm{m}$ depth to ensure optical resolution to assess the morphology of densely packed immature neurons in the GCL.

To evaluate the effect of IGF1 on numbers of cells that proliferated at $3 \mathrm{dpi}$, the number of $\mathrm{BrdU}+$ and BrdU + Dcx + cells were manually counted in the GCL, HL and the ML of the upper blade of the DG. Cells were determined to reside in the GCL if they were within one cell distance $(0-10 \mu \mathrm{m})$ of the GCL/HL border (Villasana et al., 2015). Colocalization of markers was confirmed by making $3 \mathrm{D}$ reconstructions of the cells of interest to avoid false positives. The volume $\left(\mathrm{mm}^{3}\right)$ of the respective ROI (GCL, ML, and HL) was quantified in each section, for cell counts expressed as volumetric densities. To quantify cell proliferation at $10 \mathrm{dpi}$, PCNA + cells were manually counted in GCL in one tissue section. PCNA + counts were normalized to the length of the SGZ in the upper and lower blades.

\section{Newborn Neuron Localization}

Images obtained for BrdU $+\mathrm{Dcx}+$ cell counts were used to assess cell localization within the DG. The distance from the hilar border of the GCL (GCL/HL border) to the center of the cell soma was measured for BrdU $+\mathrm{Dcx}+$ cells in the upper blade of the GCL. The inner GCL (iGCL) layer is defined as the inner 1/3rd of the GCL area (Kempermann et al., 2003; Mathews et al., 2010; Ibrahim et al., 2016). For quantification, the iGCL and SGZ cell counts ( $0-50 \mu \mathrm{m}$ from the HL) were pooled and presented as iGCL. The outer GCL (oGCL) describes the outermost $2 / 3$ of the GCL. Cells localized to the HL, further than $10 \mu \mathrm{m}$ from the GCL/HL border were considered to be ectopically localized to the HL (Villasana et al., 2015). Cell counts and distances were manually quantified.

\section{Statistical Analyses}

Graphs were generated and data were analyzed using GraphPad Prism software. Data are presented as either box and whisker plots that display the 25th, 50th, and 75th quartiles with minimum and maximum values as whiskers or as mean with error bars depicting standard error of the mean (SEM). Because areas of pS6 immunostaining in the GCL, HL, and ML were equivalent in WT and IGF1 sham controls when compared using $t$-tests, sham data were pooled for analysis. Outliers were identified using Grubbs analysis and are detailed in Supplementary Tables 1, 2. One-way analysis of variance (ANOVA) was used to assess differences among group means except as noted below. For one-way ANOVAs, data sets were evaluated using GraphPad Prism to determine whether standard deviations (SDs) were significantly different among groups. If SDs were equivalent, then a standard ANOVA with Sidak's post hoc $t$-tests was performed. If SDs were significantly different as determined using the Brown-Forsythe test, then a Welch's ANOVA was performed and Dunnett's T3 multiple comparisons tests were used for pairwise comparisons. For analysis of experiments involving rapamycin, planned post hoc comparisons were limited to four: WT Veh vs. IGFtg Veh, WT Rapa vs. IGFtg
Rapa, WT Veh vs. WT Rapa, and IGFtg Veh vs. IGFtg Rapa. Twoway ANOVA was used to assess cell localization within the GCL (Supplementary Figure 4). Two-way ANOVAs were followed by Sidak's multiple comparisons tests only when main effect interactions were found to be significant. Details of ANOVA and post hoc testing results are provided in tabular format. For all comparisons $p<0.05$ was considered statistically significant.

\section{RESULTS}

\section{IGF1 Overexpression Potentiates Injury-Induced mTOR Activation in Predominantly Neuronal Sub-regions of the DG}

To determine if posttraumatic overexpression of IGF1 amplifies injury-induced mTOR activation in the hippocampal DG, we quantified the total area of cellular immunolabeling for pS6 at 24 and $72 \mathrm{~h}$ after moderate or severe CCI (Figure 1A). Because S6 is a downstream target of mTOR activity, phosphorylation of S6 is a widely used indicator of mTOR activation (Lipton and Sahin, 2014; Wang et al., 2016; Millan et al., 2019). In control (uninjured sham) brains of both WT and IGFtg mice, only a few scattered cells exhibit S6 activation (Figure 2A). Following moderate CCI, more widespread S6 activation was noted within the GCL, especially in the IGFtg mice, which also exhibited increased pS6 in the HL. Early S6 activation in the ML was not sustained to $72 \mathrm{~h}$. Colabeling for pS6 with PCNA to detect proliferation and GFAP to detect astrocytes revealed little to no S6 activation within astrocytes at $72 \mathrm{~h}$ after moderate injury, despite some evidence of astrocyte proliferation in the ML (Figure 2B). Neuronal S6 activation within the GCL and HL was more pronounced in IGFtg mice and most pS6 + neurons were not labeled with PCNA. Quantification of pS6 staining in WT mice showed a small but statistically significant increase in the GCL and HL at $72 \mathrm{~h}$ when compared to sham controls (Figure 2C and Table 1). In contrast, IGF1 overexpressing mice exhibited significantly increased pS6 immunolabeling in all three regions at $24 \mathrm{~h}$ when compared to sham controls. This elevation was sustained to $72 \mathrm{~h}$ in the GCL and HL of IGFtg mice with moderate CCI. When compared to injured WT mice, IGF1 overexpression stimulated a significantly greater pS6 response in the GCL and HL (Figure 2C and Table 1).

Following a severe CCI, S6 activation was increased in the GCL and HL, particularly within the IGFtg mice at $72 \mathrm{~h}$ (Figure 3A). Compared to moderate CCI, severe injury initiated a notably more robust involvement of the ML at $72 \mathrm{~h}$ in both WT and IGFtg mice, with pS6 + cells exhibiting a glial morphology (Figure 3A). Colabeling with GFAP and PCNA demonstrated that $\mathrm{S} 6$ activation in the ML occurred within proliferating astrocytes in both genotypes (Figure 3B). In WT mice, severe injury stimulated a significant increase in S6 activation in the GCL at $24 \mathrm{~h}$, in the $\mathrm{HL}$ at $72 \mathrm{~h}$ and in the ML at both time points (Figure 3C and Table 1). In contrast, severely injured IGFtg mice showed significant increases in the extent of S6 activation in all three regions at both 24 and $72 \mathrm{~h}$ (Figure 3C and Table 1), 
A
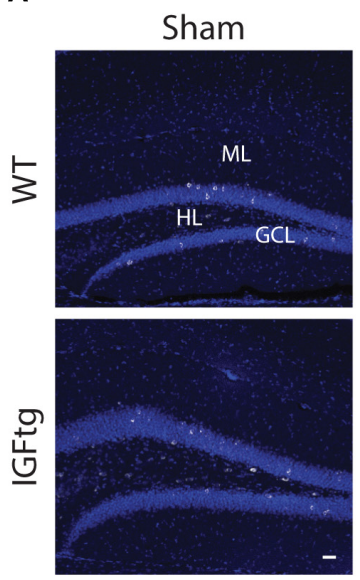

C
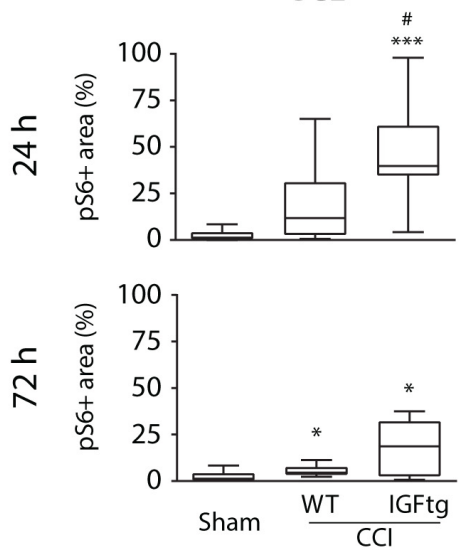

Moderate injury

$24 \mathrm{~h}$
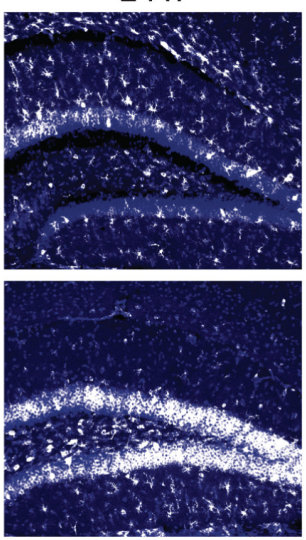

pS6 DAPI
B
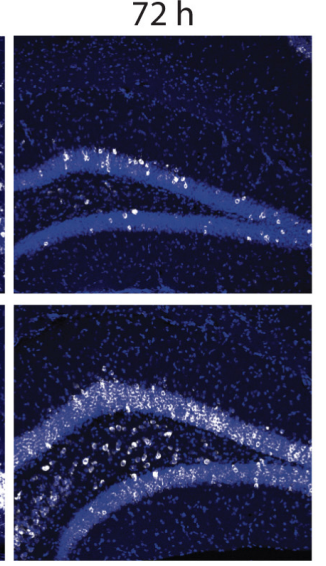

$\mathrm{HL}$

ML
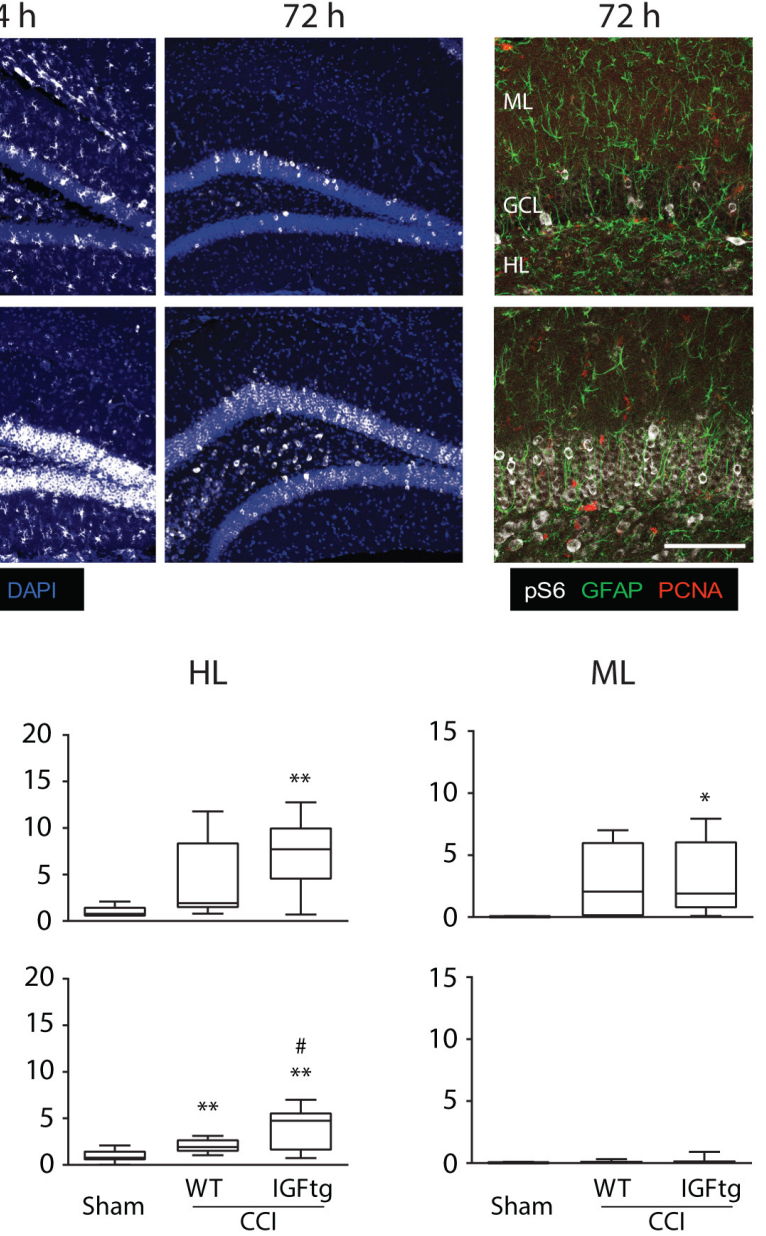

FIGURE 2 | IGF1 potentiates mTOR activity in the dentate gyrus induced by moderate brain injury. (A) Representative microphotographs from confocal imaging of immunoreactivity of phospho-S6 Ribosomal Protein (pS6, white) in wildtype (WT) and insulin-like growth factor 1 transgenic (IGFtg) mice following sham injury and 24 and $72 \mathrm{~h}$ after moderate controlled cortical impact (CCI) injury. DAPI staining is shown as blue. Scale bar represents $100 \mu \mathrm{m}$. (B) Confocal images illustrate activation of S6 predominantly in neurons at $72 \mathrm{~h}$ after moderate CCl. Astrocytes (GFAP, green) did not colabel with pS6 (white) and most were not proliferating (PCNA, red). Scale bar represents $100 \mu \mathrm{m}$. (C) The area of pS6 immunostaining was significantly increased in injured IGFtg mice when compared to either injured WT mice or Sham controls in the granule cell layer (GCL) at $24 \mathrm{~h}$ and in the hilar layer (HL) at $72 \mathrm{~h}$. In the molecular layer (ML), injury resulted in a small, transient increase in pS6 area at $24 \mathrm{~h}$, which subsided by $72 \mathrm{~h}$ post-injury. pS6 + area is presented as a percent of the region of interest area. Data are presented as quartile box plots with min-max. One-way ANOVA, followed by post hoc tests: * $p<0.05,{ }^{* *} p<0.01$, and ${ }^{* * *} p<0.001$ compared to Sham; ${ }^{*} p<0.05$ compared to WT CCI. Group sizes: Sham ( $n=12 ; 7 \mathrm{WT}, 5 \mathrm{IGFtg}), 24 \mathrm{~h} \mathrm{CCl}(n=10 \mathrm{WT}, 11 \mathrm{IGFtg}), 72 \mathrm{~h} \mathrm{CCl}(n=11 \mathrm{WT}, 11 \mathrm{IGFtg})$.

when compared to shams. As with moderate CCI, S6 activation following severe injury was augmented in IGFtg mice compared to WT mice in the GCL, a predominantly neuronal region, as well as in the HL, but not in the ML (Figure 3C and Table 1).

Interestingly, hippocampal mTOR activation after moderate and severe CCI was bilateral in injured IGFtg mice, while WT mice showed only minor S6 activation in the contralateral $\mathrm{HL}$ with severe CCI (Supplementary Figure 2A). Injured mice with IGF1 overexpression exhibited a greater area of pS6 immunolabeling within the GCL and the HL of the hippocampus contralateral to the impact than did sham-injured controls, although this response was more delayed following severe CCI (Supplementary Figure 2B and Supplementary Table 3).
The delayed mTOR signaling within astrocytes of the ML so prominent in the ipsilateral hippocampus after severe CCI in both genotypes was absent in the contralateral hippocampal ML.

\section{Delayed Administration of Rapamycin Modulates Numbers of Proliferated (BrdU+) Cells in IGFtg Mice but Not in Wildtype Mice}

We selected a dose of $10 \mathrm{mg} / \mathrm{kg}$ rapamycin based on published work related to hippocampal neurogenesis in the mouse CCI model (Butler et al., 2015; Wang et al., 2016). To validate that this dose effectively inhibited mTOR activity in our model, injured 
TABLE 1 | Statistical analysis of regional pS6 immunolabeling data from the ipsilateral hippocampal dentate gyrus.

\begin{tabular}{|c|c|c|c|c|c|c|}
\hline \multicolumn{4}{|c|}{ Moderate injury: Ipsilateral hippocampus } & \multicolumn{3}{|c|}{ Dunnett's T3 multiple comparisons test } \\
\hline Region & Timepoint & $W$-value & $p$-value & Sham vs. WT CCI & Sham vs. IGFtg CCI & WT CCl vs. GFtg CCl \\
\hline \multirow[t]{2}{*}{ GCL } & $24 \mathrm{~h}$ & 19.07 & 0.0001 & 0.078 & $\star \star \star * 0.0005$ & $\# 0.042$ \\
\hline & $72 \mathrm{~h}$ & 8.00 & 0.0034 & $\star 0.049$ & $\star 0.018$ & 0.063 \\
\hline \multirow[t]{2}{*}{$H L$} & $24 \mathrm{~h}$ & 16.36 & 0.0004 & 0.078 & $* * 0.0014$ & 0.396 \\
\hline & $72 \mathrm{~h}$ & 12.70 & 0.0004 & ${ }^{\star *} 0.0088$ & ${ }^{\star *} 0.0026$ & $\# 0.042$ \\
\hline \multirow[t]{3}{*}{ ML } & $24 \mathrm{~h}$ & 9.01 & 0.0042 & 0.066 & $\star 0.020$ & 0.982 \\
\hline & $72 \mathrm{~h}$ & 4.48 & 0.033 & 0.091 & 0.234 & 0.817 \\
\hline & \multicolumn{6}{|c|}{ Severe injury: Ipsilateral hippocampus } \\
\hline \multirow[t]{2}{*}{ GCL } & $24 \mathrm{~h}$ & 9.95 & 0.0024 & ${ }^{*} 0.049$ & ${ }^{*} 0.013$ & 0.105 \\
\hline & $72 \mathrm{~h}$ & 5.85 & 0.013 & 0.519 & $\star 0.022$ & $\# 0.041$ \\
\hline \multirow[t]{2}{*}{$H L$} & $24 \mathrm{~h}$ & 15.25 & 0.0005 & 0.069 & ${ }^{\star *} 0.0015$ & 0.675 \\
\hline & $72 \mathrm{~h}$ & 24.35 & $<0.0001$ & **0.0059 & $* \star 0.0008$ & $\# 0.023$ \\
\hline \multirow[t]{2}{*}{$\mathrm{ML}$} & $24 \mathrm{~h}$ & 11.60 & 0.0025 & *0.049 & ${ }^{\star} 0.015$ & 0.264 \\
\hline & $72 \mathrm{~h}$ & 33.15 & $<0.0001$ & ${ }^{\star \star \star *} 0.0006$ & ${ }^{* *} 0.0018$ & 0.244 \\
\hline
\end{tabular}

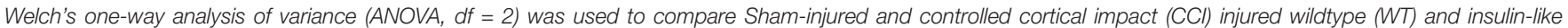

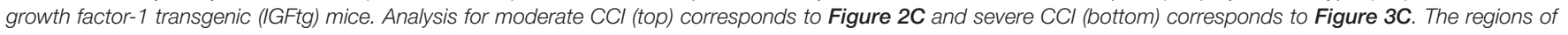

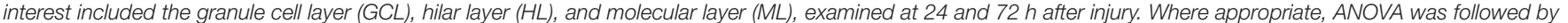

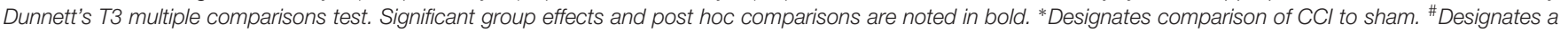
genotype effect. Number of symbols indicates level of significance, as in Figures $2 \mathrm{C}$ and $3 \mathrm{C}$.

WT and IGFtg mice were treated with daily intraperitoneal injections of rapamycin or its vehicle for 3 days beginning $1 \mathrm{~h}$ after CCI (Figure 1B). At $72 \mathrm{~h}$ after severe CCI, pS6 immunolabeling within the DG ipsilateral to impact, clearly evident in vehicle-treated mice, was effectively silenced by rapamycin treatment (Supplementary Figure 3).

Based on our findings that moderate CCI brain injury in IGFtg mice resulted in IGF1-stimulated mTOR activation more selectively in the GCL without the marked astrocytic S6 activation that accompanied severe CCI, we chose moderate CCI for studies interrogating the role of mTOR signaling in the enhancement of hippocampal neurogenesis by IGF1. Our previous work suggests that IGF1 increases posttraumatic neurogenesis by promoting neuronal differentiation of proliferating NPCs and supporting dendritic development of immature neurons born after TBI rather than by increasing proliferation (Carlson et al., 2014; Carlson and Saatman, 2018; Littlejohn et al., 2020). Therefore, to avoid rapamycin-induced inhibition of cellular proliferation (Paliouras et al., 2012; Wang et al., 2016) which would confound interpretation of neuronal differentiation and immature neuron development, we delayed rapamycin treatment until after the primary wave of proliferation. Three days after CCI, at the peak of trauma-induced proliferation (Dash et al., 2001; Rola et al., 2006), dividing cells in WT and IGFtg mice were labeled with BrdU over an $8 \mathrm{~h}$ period, after which rapamycin treatment commenced (Figure 1C).

At $10 \mathrm{dpi}$, BrdU + cells were observed clustered within the SGZ and distributed throughout the HL and ML (Figure 4A). Proliferated cells within the ML were often colabeled with the astrocyte marker GFAP, whereas far fewer phenotyped as proliferating microglia despite robust labeling with Iba1 indicative of trauma-induced microglial activation (Figure 4A). No appreciable colocalization of BrdU with the mature neuron marker NeuN was observed (data not shown). This was not unexpected as the BrdU + cells here were only 7 days old and NeuN expression begins after 1-2 weeks (Duan et al., 2008; Kernie and Parent, 2010). BrdU + cells were counted separately in the GCL, HL and the upper blade of the ML, and counts were normalized to the region volume (per section) to control for any differences in size across tissue sections or animals. In WT mice, neither the density (Figure 4B) nor the number (Figure 4C) of $\mathrm{BrdU}+$ cells was affected in any region by rapamycin treatment. These data suggest that incorporation of BrdU on day 3 and survival of proliferated cells to $10 \mathrm{dpi}$ were not notably altered by mTOR inhibition initiated after BrdU administration. Rapamycin treatment in brain-injured IGFtg mice resulted in a significant increase in both the density and number of proliferated cells in the GCL (Figures 4B,C and Table 2). In contrast, the density of proliferated cells in the ML was notably decreased in the IGFtg mice that received rapamycin (Figures 4A,B). Examination of the relative densities of BrdU + cells in these two regions, the GCL and ML, showed that the mean density of BrdU + cells in the GCL was 1.2 times that in the ML in vehicle-treated WT mice, while the GCL:ML ratio was 3.1 in IGFtg mice. In rapamycin-treated mice, IGF1 overexpression resulted in a sevenfold increase in the GCL density: ML density ratio, from 1.4 in WT mice to 9.5 in IGFtg mice. Proliferation within the HL was not altered by IGF1 overexpression or inhibition of mTOR signaling.

\section{IGF1-Mediated Hippocampal Neurogenesis Is Not Diminished by Rapamycin Administration}

To determine whether mTOR signaling is important for IGF1mediated cell fate determination of posttrauma-proliferated 
A

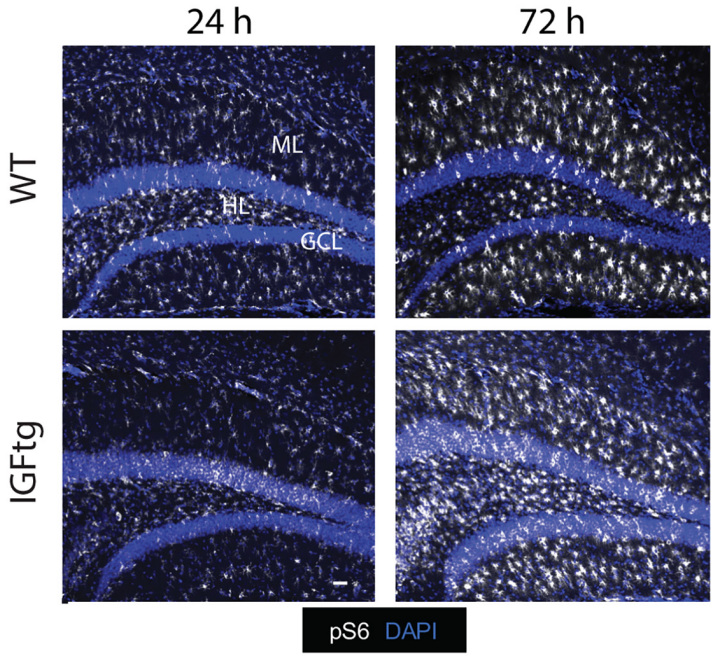

B

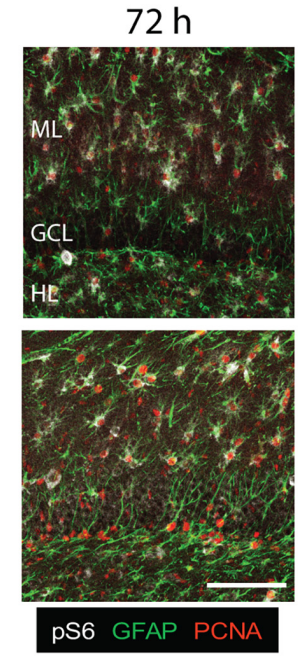

C

GCL

$\mathrm{HL}$

$\mathrm{ML}$
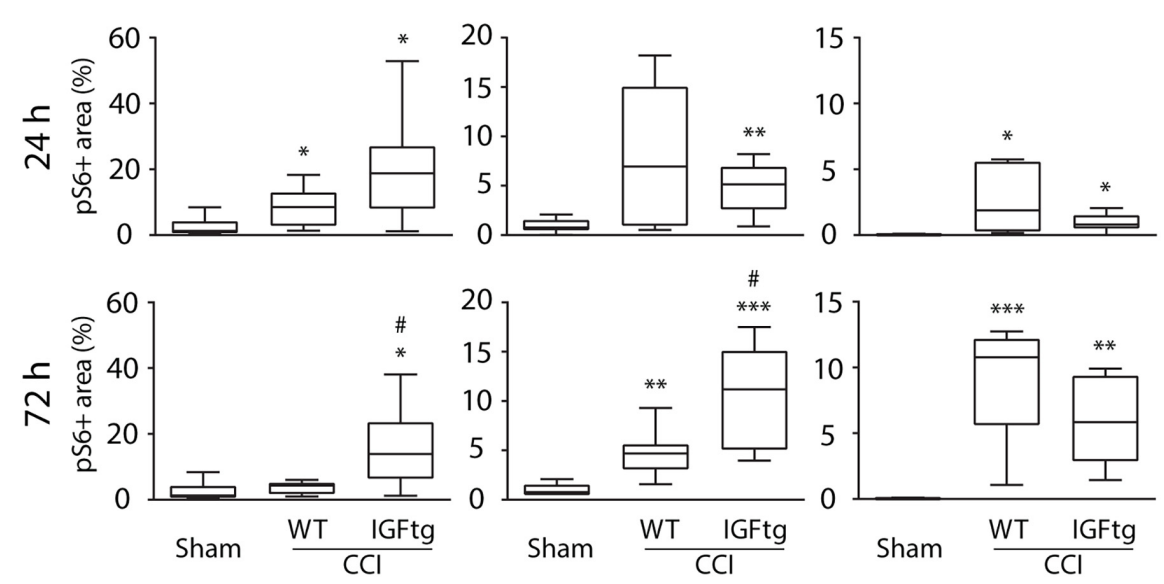

FIGURE 3 | IGF1 selectively enhances granule cell and hilar layer mTOR activity, without affecting delayed S6 activation in proliferating astrocytic in the molecular layer after severe injury. (A) Representative confocal images of immunoreactivity of phospho-S6 Ribosomal Protein (pS6, white) in wildtype (WT) and insulin-like growth factor 1 transgenic (IGFtg) mice following sham injury and 24 and $72 \mathrm{~h}$ after severe controlled cortical impact (CCI) injury. DAPI staining is shown as blue. Scale bar represents $100 \mu \mathrm{m}$. (B) Confocal images illustrating colocalization of pS6 (white) and proliferating cell nuclear antigen (PCNA, red) within astrocytes (GFAP, green) of the molecular layer (ML) in the hippocampus ipsilateral to impact at $72 \mathrm{~h}$ after severe $\mathrm{CCl}$. Scale bar represents $100 \mathrm{\mu m}$. (C) In the granule cell layer (GCL) and the hilar layer (HL), IGFtg mice exhibited a significantly larger area of pS6 staining when compared to either injured WT mice or sham controls at $72 \mathrm{~h}$ after CCl. In the ML, both WT and IGFtg mice exhibited significant increases in pS6 levels at $72 \mathrm{~h}$. pS6 + area is presented as a percent of the region of interest area. Data are presented as quartile box plots with min-max. One-way ANOVA, followed by post hoc tests: * $p<0.05$, ${ }^{* *} p<0.01$, and *** $p<0.001$ compared to Sham; ${ }^{\#} p<0.05$ compared to WT CCI. Group sizes: Sham ( $n=12 ; 7$ WT, 5 IGFtg), 24 h CCI ( $n=9$ WT, 10 IGFtg), 72 h CCI $(n=9$ WT, 10 IGFtg).

progenitors, we identified cells labeled with BrdU at 3 days after TBI (prior to the onset of rapamycin treatment) that phenotyped as immature neurons (Dcx + ) at $10 \mathrm{dpi}$ (Figure 5A). IGF1 overexpression was associated with a significant, nearly twofold, increase in the density of BrdU + immature neurons in the GCL of vehicle-treated mice (Figure 5B and Table 2). Inhibition of mTOR signaling did not prevent stimulation of neurogenesis by IGF1, but appeared to augment the number of posttrauma-proliferated cells within the GCL that committed to a neuronal fate in IGFtg mice, although this increase did not reach statistical significance ( $p=0.062$, Figure 5B). Because rapamycin treatment in IGFtg mice resulted in higher numbers of BrdU + cells present in the GCL at $10 \mathrm{dpi}$, we also examined the numbers of newly born neurons as a proportion of all BrdU-labeled cells. Only approximately $40 \%$ of cells proliferating at 3 dpi phenotyped as immature GCL neurons at $10 \mathrm{dpi}$ in WT mice, regardless of treatment, whereas rapamycin treatment significantly increased this proportion to nearly $80 \%$ in IGFtg mice (Figure 5C and Table 2). These data demonstrate that IGF1 enhances acute posttraumatic neurogenesis and that delayed rapamycin treatment in combination with IGF1 overexpression increases the proportion of proliferated cells that commit to 
A
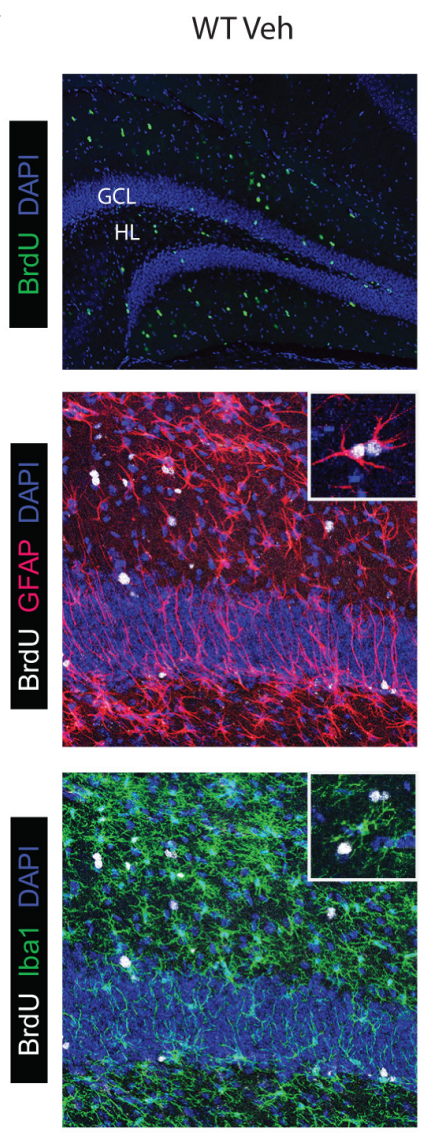

B

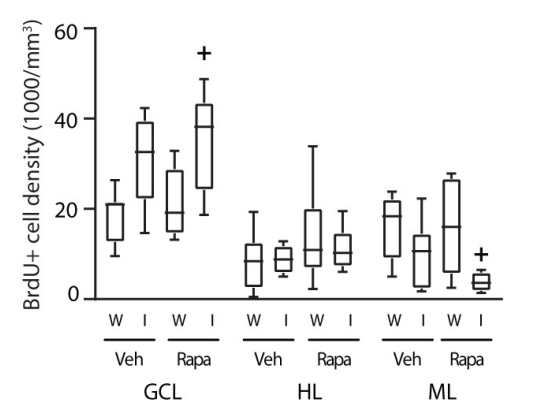

IGFtg Veh
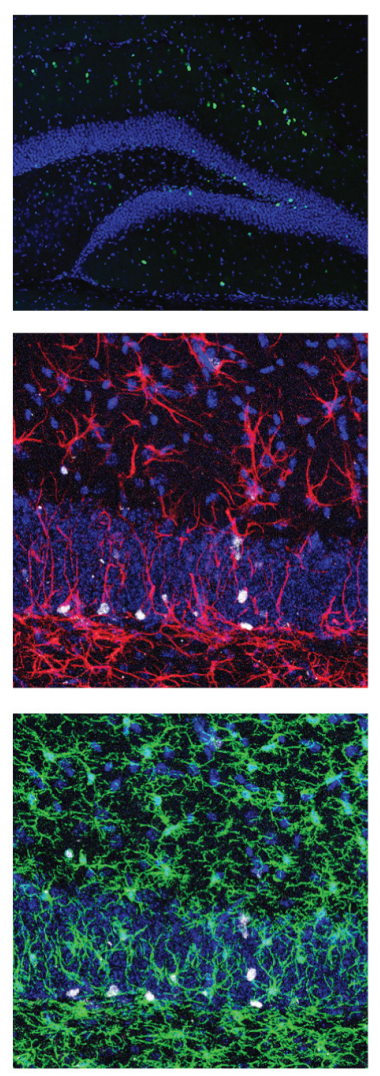

C

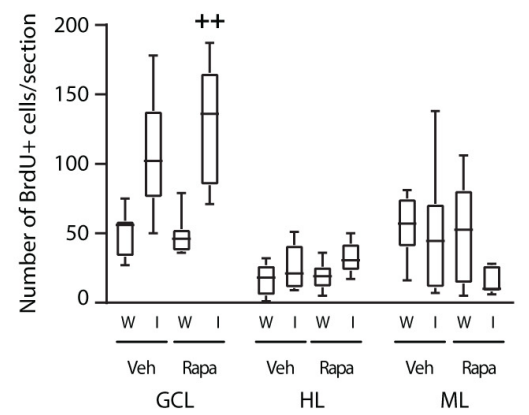

WT Rapa
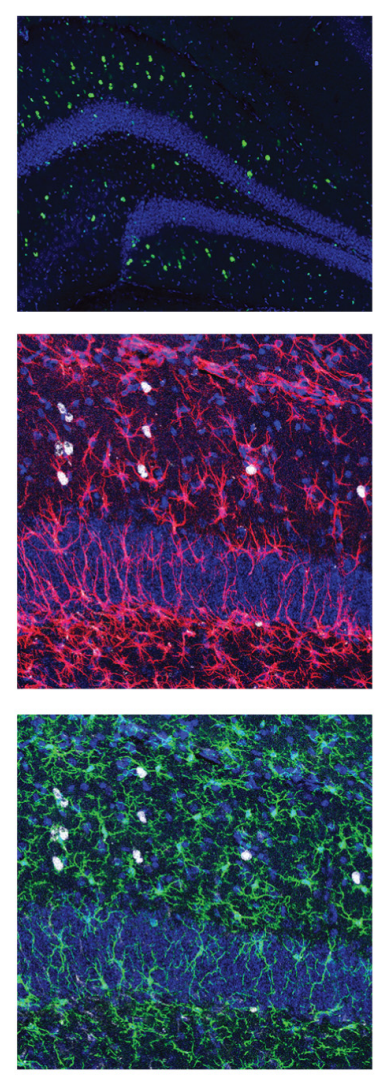

IGFtg Rapa
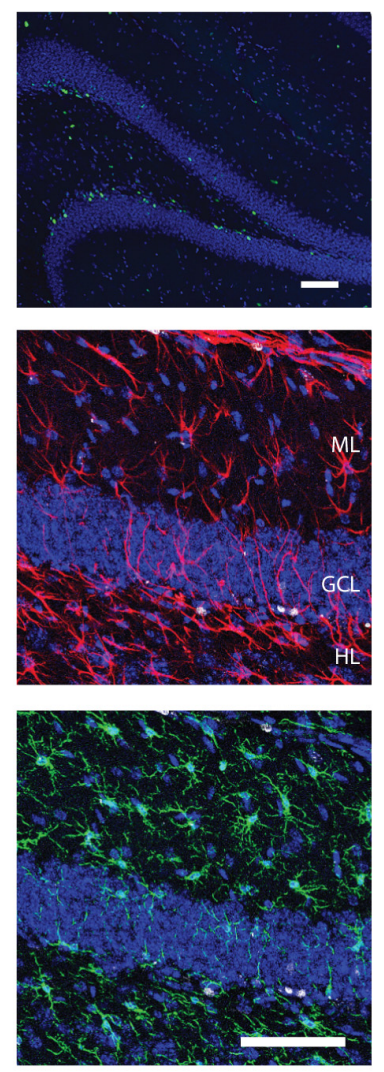

D

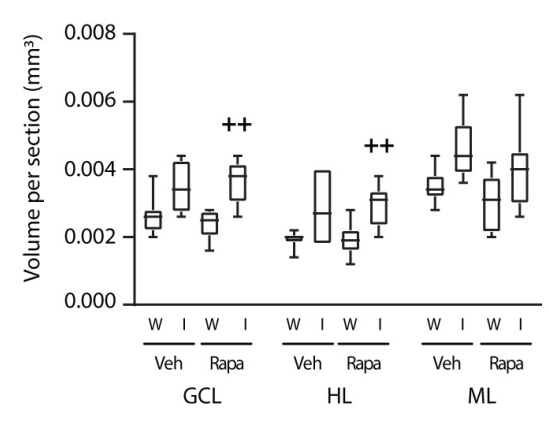

FIGURE 4 | IGF1 overexpression in combination with mTOR inhibition increases the density of proliferated cells in the granule cell layer while decreasing proliferation in the molecular layer. (A) Top row: Traumatic brain injury results in cellular proliferation (bromodeoxyuridine; BrdU, green) throughout the ipsilateral dentate gyrus of wildtype (WT, W) and IGF1 transgenic (IGFtg, I) mice that received vehicle (Veh) or rapamycin (Rapa). Middle, bottom rows: IGFtg mice had more proliferated cells in the granule cell layer (GCL) but fewer within the molecular layer (ML) when compared to WT mice. Colabeling of BrdU (white) with GFAP (red) or lba1 (green) demonstrated that cells dividing at 3 dpi more frequently phenotyped as astrocytes than microglia in the ML (see insets). DAPI staining is shown as blue. Scale bar represents $100 \mu \mathrm{m}$. (B) At 10 days after moderate injury, proliferated cells (BrdU +) in the GCL, ML and hilar layer (HL) were quantified. In IGFtg mice treated with Rapa the density (cell number/volume) of BrdU + cells in the GCL was increased while it was decreased in the ML. (C) Analysis of non-normalized cell counts revealed increased numbers of BrdU + cells in the GCL of Rapa-treated IGFtg mice. (D) GCL and HL volumes were significantly greater in IGFtg mice receiving rapamycin than in similarly treated WT mice. Data are represented as quartile box plots with min-max. One-way ANOVA, followed by post hoc tests: $+p<0.05$ and $++p<0.01$ compared to WT Rapa. Group sizes: WT Veh $n=7$, IGFtg Veh $n=6$, WT Rapa $n=8$, IGFtg Rapa $n=8$

a neuronal phenotype after TBI. In addition to supporting an increase in the density of immature neurons after TBI, IGF1 overexpression was associated with improved dendritic architecture within Dcx + cells (Figures 5A, 6A), replicating our previous work establishing a significant enhancement of immature neuron dendrite length and complexity in mice with IGF1 overexpression (Carlson et al., 2014). Rapamycin treatment appeared to inhibit dendrite development in brain-injured WT 
mice; however, this inhibitory effect was not notable in IGFtg mice (Figures 5A, 6A).

\section{Positioning of Posttrauma-Born Immature GCL Neurons Is Not Altered by Rapamycin}

Brain injury has been reported to increase outward migration of immature neurons from the SGZ (Villasana et al., 2015; Ibrahim et al., 2016; Shapiro, 2017). Regulation of the positioning of adultborn neurons in the uninjured brain may involve endogenous IGF1 and mTOR signaling pathways (Zhu et al., 2012; NietoEstevez et al., 2016), and we have shown that IGF1 overexpression supports greater outward migration of posttrauma-born neurons in the GCL while reducing the proportion mislocalized to the HL (Littlejohn et al., 2020). Examination of the location of new neurons within the GCL revealed, as expected, that the vast majority of 7-day old neurons were found in the SGZ and iGCL for all groups, with 95\% or greater in WT mice (Figure 5D). IGF1 overexpression stimulated a small but significant increase in radial migration into the oGCL at $10 \mathrm{dpi}$ (Table 2). Rapamycin did not affect immature neuron migration in either WT or IGFtg injured mice. Although the proportion of newly born GCL neurons within the iGCL did not change with rapamycin treatment (Figure 5D), mTOR inhibition in IGFtg mice resulted in significantly greater numbers of BrdU $+\mathrm{Dcx}+$ cells within the first 20 microns from the SGZ/HL border as compared to IGFtg mice receiving vehicle and within the first 40 microns when compared to rapamycin-treated WT mice (Supplementary Figure 4). Only a very small number of 7-day old posttraumaborn immature neurons localized to the HL following moderate
$\mathrm{CCI}$, and the proportion of ectopically localized new neurons was not different across groups (Figure 5E).

\section{Daily Rapamycin Administration Does Not Impair GCL Proliferation at $\mathbf{1 0} \mathrm{dpi}$}

To determine whether daily administration of rapamycin resulted in suppression of SGZ proliferation in the subacute period after CCI, brain sections were immunolabeled for Dcx and PCNA. In stark contrast to the substantial wave of proliferation in the ML of WT mice captured by BrdU administration at 3 dpi (see Figure 4), at 10 dpi proliferating cells were localized primarily within the SGZ, with few PCNA + cells in the ML (Figure 6A). The density of proliferating cells within the GCL was not significantly altered by either IGF1 overexpression or rapamycin administration (Figure 6B and Table 2).

\section{DISCUSSION}

This study aimed to determine if pro-neurogenic effects of IGF1 in the injured brain are dependent upon mTOR activation. By mapping S6 activation in the hippocampal DG through immunolabeling, we show that conditional, astrocyte-specific IGF1 overexpression selectively modulated regional patterns of posttraumatic mTOR signaling, leading to greater activation within the GCL and HL in the first 3 days after injury. Inhibition of mTOR by rapamycin, however, did not abrogate the enhanced neurogenesis observed in IGFtg mice after CCI brain injury. Rather, mTOR inhibition in mice with elevated brain levels of IGF1 resulted in an increased density of BrdU + cells within the GCL and an increase in the proportion of proliferated cells that differentiated into GCL neurons by $10 \mathrm{dpi}$. These data

TABLE 2 | Statistical analysis of posttraumatic proliferation, neuronal differentiation, and positioning in the dentate gyrus.

\begin{tabular}{|c|c|c|c|c|c|c|c|}
\hline \multicolumn{4}{|c|}{ Neurogenesis measures } & \multicolumn{4}{|c|}{ Post hoc comparisons } \\
\hline Measure & Figure & $F / W$-value & $p$-value & WT vs. IGFtg (Veh) & WT vs. IGFtg (Rapa) & Veh vs. Rapa (WT) & Veh vs. Rapa (IGFtg) \\
\hline BrdU + density (GCL) & $4 \mathrm{~B}$ & 5.85 & 0.0038 & 0.083 & +0.020 & 0.967 & 0.798 \\
\hline BrdU + density (HL) & $4 \mathrm{~B}$ & 0.93 & 0.442 & - & - & - & - \\
\hline BrdU + density (ML) & $4 \mathrm{~B}$ & 9.02 & 0.0028 & 0.495 & +0.046 & 1.000 & 0.288 \\
\hline BrdU + cell count (GCL) & $4 \mathrm{C}$ & 10.45 & 0.0011 & 0.076 & ++0.0026 & 1.000 & 0.788 \\
\hline BrdU + cell count $(H L)$ & $4 \mathrm{C}$ & 2.31 & 0.103 & - & - & - & - \\
\hline BrdU + cell count (ML) & $4 \mathrm{C}$ & 2.33 & 0.100 & - & - & - & - \\
\hline GCL volume & $4 \mathrm{D}$ & 8.35 & 0.0005 & 0.098 & ++0.007 & 0.785 & 0.947 \\
\hline HL volume & $4 \mathrm{D}$ & 6.70 & 0.0063 & 0.244 & ++0.0096 & 1.000 & 0.998 \\
\hline ML volume & $4 \mathrm{D}$ & 4.00 & 0.019 & 0.109 & 0.155 & 0.854 & 0.629 \\
\hline BrdU + Dcx + density (GCL) & $5 B$ & 9.93 & 0.0010 & \#\#0.0067 & +0.015 & 0.808 & 0.062 \\
\hline BrdU + Dcx + : BrdU (GCL) & $5 \mathrm{C}$ & 8.59 & 0.0005 & 0.735 & +++0.0006 & 1.000 & ${ }^{\circledR} 0.021$ \\
\hline iGCL: GCL (BrdU + Dcx +) & $5 \mathrm{D}$ & 3.20 & 0.042 & $\# 0.047$ & 0.500 & 0.920 & 0.910 \\
\hline HL: DG (BrdU + Dcx +) & $5 E$ & 1.79 & 0.204 & - & - & - & - \\
\hline PCNA + density (GCL) & $6 B$ & 1.12 & 0.360 & - & - & - & - \\
\hline
\end{tabular}

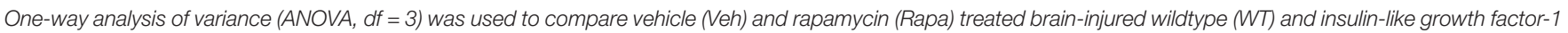

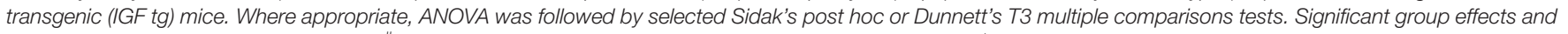

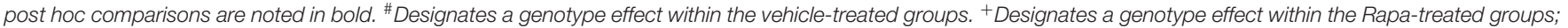

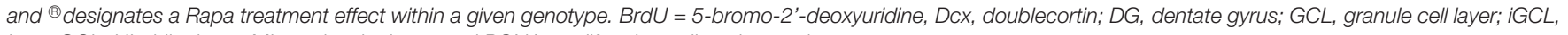
inner GCL; HL, hilar layer; ML, molecular layer; and PCNA, proliferating cell nuclear antigen. 
A
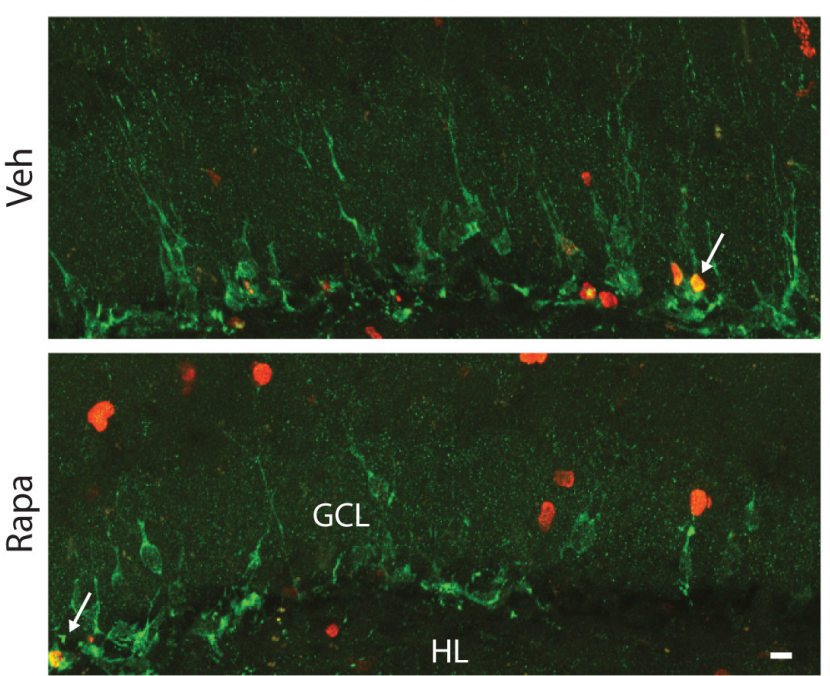

8

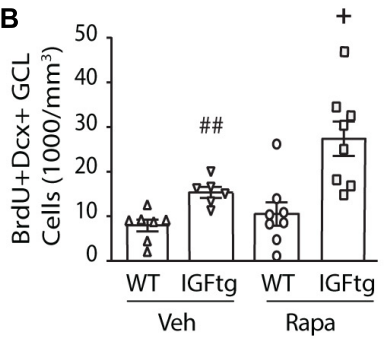

C

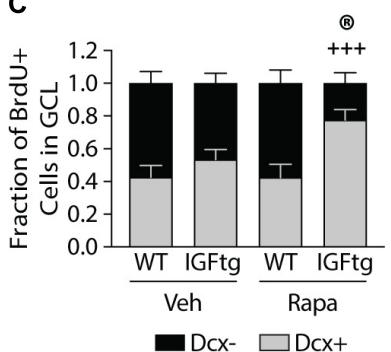

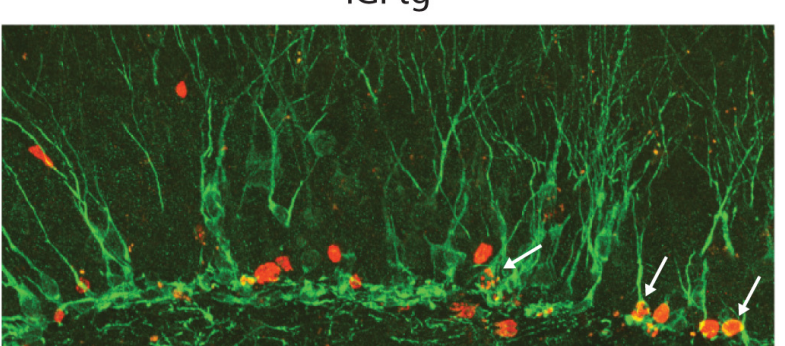

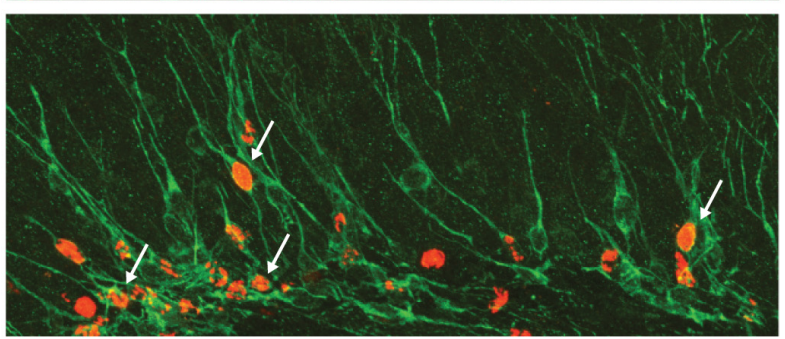

D

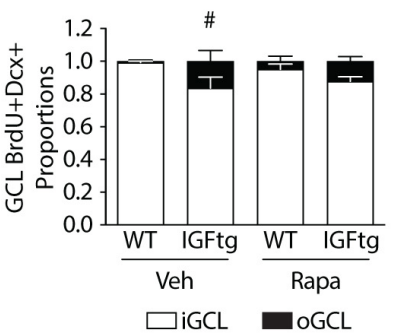

E

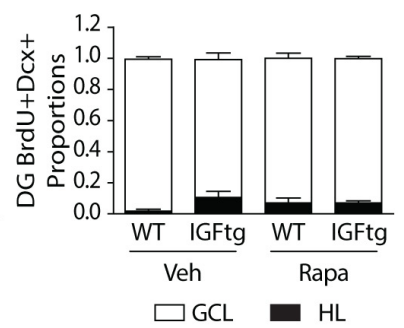

FIGURE 5 | The density and outward migration of posttrauma-born granule neurons is increased in IGF1 overexpressing mice. (A) Representative images of immunofluorescence colocalization of doublecortin (Dcx, green), a marker for immature neurons, and the proliferation marker BrdU (red) in the granule cell layer (GCL) of vehicle (Veh) and rapamycin (Rapa) treated wildtype (WT) and IGF1 transgenic (IGFtg) mice at 10 days after injury. White arrows mark double labeled cells. Scale bar represents $20 \mu \mathrm{m}$. (B) The density of BrdU + Dcx + neurons in the GCL was significantly greater in IGFtg mice compared to their treatment-matched WT groups. (C) Rapamycin treatment of IGFtg mice injected with BrdU at 3 dpi resulted in a significantly higher proportion of proliferated cells that differentiated into neurons by $10 \mathrm{dpi}$. (D) The proportion of immature neurons localized to the outer two-thirds of the GCL (oGCL) was increased in vehicle-treated IGFtg mice when compared to WT mice. Inhibition of mTOR activity did not alter migration within the GCL. (E) The proportion of newly born dentate gyrus neurons localized to the hilus $(\mathrm{HL})$ at 10 days after moderate injury was not altered by IGF1 overexpression or Rapa treatment. Data are presented as mean + SEM, with individual data points in (B). In Figures C-E, proportions per region are stacked for reference. One-way ANOVA, followed by Sidak's post hoc tests: ${ }^{\#} p<0.05$ and $\# \#<0.01$ compared to WT Veh; ${ }^{+} p<0.05$ and ${ }^{+++} p<0.001$ compared to WT Rapa; ${ }^{\circledR} p<0.05$ compared to IGFtg Veh. Group sizes: WT Veh $n=7$, IGFtg Veh $n=6$, WT Rapa $n=8$, IGFtg Rapa $n=8$.

provide new insights into the mechanisms of IGF1-mediated neurogenesis in the context of TBI.

\section{mTOR Activation}

Activation of the mTOR pathway has been established in a variety of rodent models of TBI. Rapid increases in hippocampal pS6 levels, assessed by Western blotting, peak between $30 \mathrm{~min}$ to 1 day after injury, before returning to baseline by 3 days (Park et al., 2012; Gibb et al., 2015; Wang et al., 2016) or as late as 2 weeks after CCI in mice (Guo et al., 2013). Activation of mTOR signaling in the DG is first observed in neurons, while reactive astrocytes and microglia within the ML contribute to increased pS6 labeling in a delayed manner, at 24-72 h (Chen et al., 2007; Zhu et al., 2014; Gibb et al., 2015; Nikolaeva et al., 2016; Wang et al., 2016). Our data in WT mice is consistent with these previous studies, and is the first study to our knowledge to directly compare hippocampal mTOR activation patterns as a function of injury severity and to implicate mTOR signaling in posttraumatic astrocyte proliferation in the hippocampus. Moderate CCI resulted in only a small degree of S6 activation across the DG of WT mice, while severe CCI produced a progressive increase in ML mTOR signaling which involved proliferating astrocytes. Inhibition of mTOR initiated within the first few hours after TBI has been shown to be neuroprotective and to reduce reactive astrocytosis and microgliosis (Erlich et al., 2007; Guo et al., 2013; Ding et al., 2015; Song et al., 2015; Nikolaeva et al., 2016). Our findings raise the possibility that acute rapamycin administration may reduce reactive gliosis, in part, through suppression of glial proliferation. Indeed, rapamycin has been shown to effectively reduce astrocyte proliferation in the context of hypoxic/ischemic injury and spinal cord injury (Goldshmit et al., 2015; He et al., 2019). However, further studies are warranted to elucidate the specific functions of neuronal and glial mTOR signaling in TBI. It is likely that we did not capture maximal neuronal S6 activation in WT mice, which 


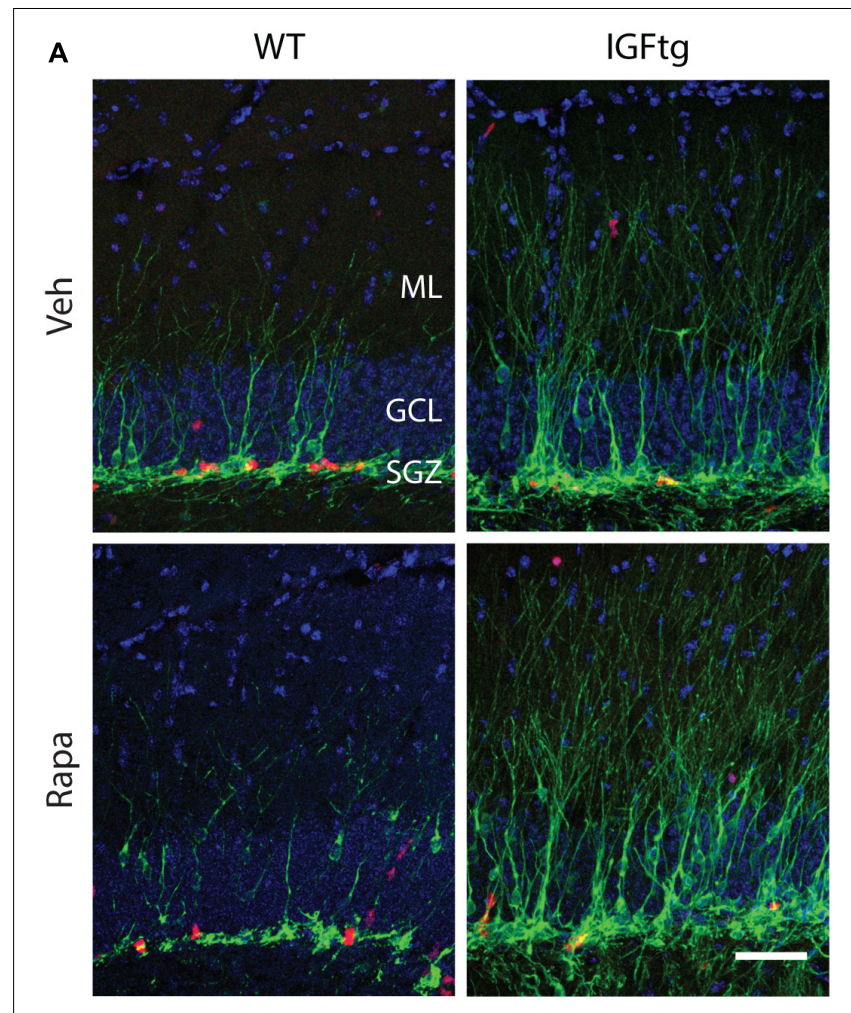

B

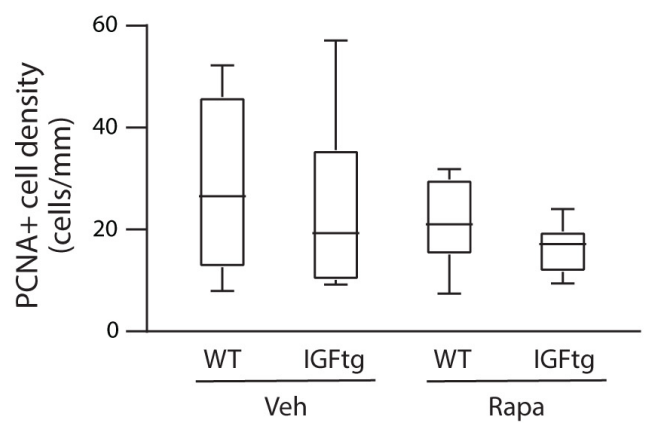

FIGURE 6 | Cellular proliferation at 10 days after injury is not altered by IGF1 overexpression or administration of rapamycin. (A) Representative images of doublecortin (Dcx, green) and proliferating cell nuclear antigen (PCNA, red) immunolabeling of the dentate gyrus in wildtype (WT) and IGF1 transgenic (IGFtg) mice that received vehicle (Veh) or rapamycin (Rapa). Inhibition of mTOR signaling does not notably diminish proliferation in the SGZ at 10 dpi for either genotype. DAPI staining is shown as blue. Scale bar represents $100 \mu \mathrm{m}$. (B) Counts of PCNA + cells within the granule cell layer (GCL), normalized to the length of the SGZ in each tissue section, were equivalent across all groups. Data are represented as quartile box plots with min-max. One-way ANOVA, $p=$ n.s. Group sizes: WT Veh $n=7$, IGFtg Veh $n=6$, WT Rapa $n=8$, IGFtg Rapa $n=8$.

may occur prior to $24 \mathrm{~h}$ postinjury. The current study focused on 24 and $72 \mathrm{~h}$ postinjury because during this period hippocampal IGF1 levels are increasing after CCI in this IGFtg mouse model (Madathil et al., 2013) and SGZ proliferation is at its peak (Dash et al., 2001; Rola et al., 2006; Bye et al., 2011).
Overexpression of IGF1 resulted in a more pronounced and widespread activation of S6 in the hippocampus of mice with CCI. The extent of pS6 immunostaining in both the GCL and HL of brain-injured IGFtg mice was significantly increased compared to either sham controls or injured WT mice. IGF1-enhanced mTOR activation in the GCL and HL was evident at both time points after moderate and severe CCI, and was detected bilaterally. This pattern is in clear contrast with S6 activation within the ML, which occurred in a delayed fashion following severe CCI, was largely restricted to the ipsilateral hippocampus, was predominantly localized to proliferating astrocytes, and was not augmented in IGFtg mice. These data suggest that astrocytespecific IGF1 overexpression stimulated a bilateral activation of neuronal mTOR signaling within the GCL and HL after TBI. In the IGFtg model used here, reactive astrocytes, by way of higher GFAP transcription, drive production of human IGF1 (Ye et al., 2004; Madathil et al., 2013). Human IGF1 protein levels were found to be elevated bilaterally in the hippocampus and to increase from 24 to $72 \mathrm{~h}$ after CCI; however, levels were significantly higher in the ipsilateral hippocampus, in keeping with greater astrogliosis ipsilateral to the impact (Madathil et al., 2013). These data raise the possibility that even a low level of IGF1 is sufficient to maximally activate S6 in the GCL and HL, as S6 activation in these regions was not notably greater in the ipsilateral than contralateral hippocampus, at $72 \mathrm{~h}$ than $24 \mathrm{~h}$, or with severe compared to moderate CCI. It is worth noting that in both WT and IGFtg mice, pS6 immunolabeled cells were located throughout the inner and outer layers of the GCL following injury, as noted by others (Chen et al., 2007; Nikolaeva et al., 2016). Preferential pS6 staining within SGZ NPCs reported at $24 \mathrm{~h}$ after CCI is transient and was not observed at 4 or $48 \mathrm{~h}$ (Wang et al., 2016). It is possible that we did not detect this transient activation due to slight differences in the injury model across labs contributing to a shift in the timing of S6 activation in SGZ NPCs.

\section{Early Proliferation and Acute Survival}

Brain injury stimulates proliferation throughout all layers of the DG, peaking on the order of 2-3 days following injury (Dash et al., 2001; Rola et al., 2006; Bye et al., 2011; Gao and Chen, 2013). While the majority of proliferating cells are glial cells located outside the GCL, proliferation is also increased in the SGZ, resulting in the generation of new granule neurons (Kernie et al., 2001; Sun et al., 2005; Gao and Chen, 2013; Wang et al., 2016). By delaying rapamycin administration until after BrdU incorporation on day 3 after injury, we avoided suppression of early posttraumatic proliferation of NPCs (Wang et al., 2016) and astrocytes (He et al., 2019) by rapamycin, as demonstrated by equivalent BrdU + cell counts in vehicle- and rapamycin-treated WT mice across all subregions of the DG. In mice overexpressing IGF1, rapamycin treatment resulted in more BrdU + cells detected in the GCL and fewer in the ML. IGF1 overexpression alone resulted in a similar trend toward higher BrdU + cell numbers, suggesting a mild effect on either proliferation at $3 \mathrm{dpi}$ or enhanced survival of proliferated cells to $10 \mathrm{dpi}$ within the GCL. IGF1 has been shown to stimulate NPC proliferation in vitro as well as in vivo, in both uninjured 
and ischemic rats (Aberg et al., 2003; Dempsey et al., 2003). However, other studies suggest that IGF1 promotes neurogenesis by enhancing neuronal differentiation rather than proliferation (Arsenijevic and Weiss, 1998; McCurdy et al., 2005; Carlson et al., 2014; Littlejohn et al., 2020). It is possible that rapamycin, which has shown to be neuroprotective in TBI, when combined with IGF1, acts to increase survival of proliferated cells within the GCL, leading to a significant increase in GCL BrdU + cell density. Inhibition of mTOR has been shown to reduce astrocyte proliferation (Goldshmit et al., 2015; He et al., 2019), which could contribute to a decrease in ML proliferation in rapamycintreated mice. However, this regional decline was only noted in the IGFtg mice. As IGF1 is thought to support astrocyte and microglial proliferation (O'Kusky and Ye, 2012), the mechanism underlying decreased proliferation in the ML in IGFtg mice receiving rapamycin requires further investigation.

One caveat to consider in interpreting our BrdU data is that contusion TBI is associated with a transient opening of the BBB throughout the contused cortex, which peaks within an hour in the cortex and diminishes over $24 \mathrm{~h}$ postinjury (Bharadwaj et al., 2016). Hippocampal BBB breakdown may also occur at higher levels of injury. Opening of the BBB could influence local BrdU concentrations and cellular uptake in the brain if $\mathrm{BrdU}$ is not administered at saturating concentrations (Taupin, 2007). We expect that this potential confound is minimal in our study for several reasons: (1) We compared proliferation across groups of injured mice, not between sham and injured mice; (2) Although $200-300 \mathrm{mg} / \mathrm{kg}$ is recommended as a saturating dose based on rat studies (Taupin, 2007), the $50 \mathrm{mg} / \mathrm{kg}$ dose we employed has been shown to label on the order of $90 \%$ of S-phase proliferating cells in the DG (Burns and Kuan, 2005) and has been used in multiple studies of hippocampal neurogenesis in mice, including those involving TBI (Kempermann et al., 2003; Rola et al., 2006; Shi et al., 2007; Gao et al., 2009; Peters et al., 2018); (3) We used a moderate level of CCI which is associated with minimal hippocampal BBB disruption (Orhan et al., 2016; Yu et al., 2019) and administered BrdU in a delayed fashion ( 3 dpi). Mice administered $50 \mathrm{mg} / \mathrm{kg}$ BrdU 3 times on day 2 after CCI did not show increased BrdU labeling within the GCL when compared to sham controls (Peters et al., 2018).

\section{Generation of Newborn Neurons}

TBI causes a loss of immature hippocampal neurons in the GCL over several days, which is followed by spontaneous recovery over 1-2 weeks, supported by increased proliferation of NPCs in the SGZ (Kernie et al., 2001; Rola et al., 2006; Yu et al., 2008; Carlson et al., 2014). Using the same IGFtg mouse model as in the current study, we previously showed that while astrocytedriven overexpression of IGF1 did not protect against early TBI-induced loss of immature neurons, it significantly increased the numbers of neurons born within the first week after CCI that phenotyped as immature neurons at 10 days (Carlson et al., 2014) and as mature GCL neurons at 6 weeks (Littlejohn et al., 2020). The ability of IGF1 to improve recovery of the immature neuron population was confirmed using intracerebroventricular infusion of IGF1 in mice with CCI (Carlson and Saatman, 2018). In the current study, we provide independent replication of our previous findings, demonstrating that IGF1 overexpression resulted in an increased density of newborn neurons in the GCL in brain-injured mice.

Numerous lines of evidence support a role for IGF1 signaling in stimulating neuronal differentiation (Arsenijevic and Weiss, 1998; Aberg et al., 2000; McCurdy et al., 2005; Otaegi et al., 2006). Rapamycin treatment attenuates hippocampal NPC differentiation stimulated by both IGF1 and insulin (Han et al., 2008; Zhang et al., 2014), implicating mTOR as a downstream mediator. Indeed, inhibition of mTORC1 activity using an shRNA approach resulted in decreased numbers of newborn SVZ neurons, while excessive activation of mTORC1 resulted in increased neuronal differentiation without an effect on proliferation (Hartman et al., 2013). Therefore, we hypothesized that rapamycin treatment would block IGF1-stimulated increases in newborn neuron numbers after injury. Interestingly, inhibiting mTOR activation did not attenuate the neurogenic potential of IGF1, but rather appeared to further enhance it, with an effect size nearing statistical significance $(p=0.06)$. Rapamycin treatment in IGFtg mice altered differentiation of cells proliferating at $3 \mathrm{dpi}$, as indicated by an increase in the proportion of BrdU + cells that expressed the immature neuron marker Dcx at $10 \mathrm{dpi}$. This study provides novel evidence that, in the context of traumatic injury, mTOR provides negative feedback on neuronal differentiation downstream of the IGF1 pathway.

Under conditions of prolonged stimulation of IGF1 or insulin receptors, PI3K and Akt activation result in phosphorylation and eventual degradation of insulin receptor substrate-1 (IRS1) as a means of negative feedback control (Haruta et al., 2000; Harrington et al., 2005). Akt activation in response to IGF1 receptor binding activates mTORC1, which in turn inhibits Akt, thereby limiting IGF1-mediated actions (O'Reilly et al., 2006; Chen et al., 2010). Rapamycin, by inhibiting mTORC1, releases these negative feedback mechanisms, resulting in increased pAkt and IRS-1 (O'Reilly et al., 2006; Carracedo et al., 2008; Carloni et al., 2010). Our data suggest that posttraumatic rapamycin administration, in IGFtg mice with prolonged elevation of brain IGF1 levels, releases negative feedback on IGF1 signaling, resulting in enhanced neurogenesis through increased proliferation and neuronal differentiation.

In brain-injured WT mice, delayed onset inhibition of mTOR signaling did not affect newborn neuron density in the GCL. To our knowledge, this is the first demonstration that mTOR activation is not required for neuronal differentiation of proliferated NPCs in the context of TBI. Rapamycin treatment initiated within 20-30 min and continuing for several days after CCI in mice has been shown to block TBI-induced increases in immature neuron numbers in the GCL (Butler et al., 2015). This effect, however, likely stems from inhibition of NPC proliferation, given that rapamycin treatment spanning the first 2 days after TBI effectively suppresses posttraumatic NPC proliferation (Wang et al., 2016).

Our studies do not distinguish effects of IGF1 or rapamycin on specific developmental stages of hippocampal NPCs and neuronal progenitor cells. The neurogenic niche of the SGZ contains quiescent, radial-glia-like NPCs which divide asymmetrically, giving rise to amplifying NPCs that then 
undergo rapid symmetric division to generate neuroblasts (Plumpe et al., 2006; Encinas and Enikolopov, 2008; Kernie and Parent, 2010). Doublecortin expressing neuroblasts represent committed neuronal precursors, but at early stages retain proliferative capacity and exhibit a bipolar morphology, typically oriented horizontally within the SGZ. In contrast, postmitotic, Dcx + neuroblasts, or immature neurons, extend an apical dendrite transversely through the GCL, branching within the ML. Although increased SGZ proliferation in response to TBI is well established, few studies have examined the effects of TBI on specific subsets of NPCs and neuronal precursor cells. Yu et al. (2008) used nestin-GFP transgenic mice to establish that CCI increases proliferation of quiescent NPCs in the SGZ by 3 days, while an increase in proliferation of $\mathrm{Dcx}+$ neuronal precursor cells occurs later, at $7 \mathrm{dpi}$. Using rigorous cell counting methodology, Gao et al. (2009) corroborated that TBI stimulates proliferation of quiescent rather than amplifying NPCs.

\section{Migration and Dendritic Development}

Adult-born, immature neurons position within 1-2 weeks of their birth in the uninjured brain (Kuhn et al., 1996; Kempermann et al., 2003). While during embryonic development hippocampal NPCs give rise to neurons that localize preferentially in the outer third of the GCL, the majority of neurons born within the SGZ of adults migrate only a short distance and reach maturity in the iGCL (Kempermann et al., 2003; Mathews et al., 2010). Traumatic injury to the brain disturbs the normal pattern of adult-born neuron migration, resulting in increased numbers of immature neurons localized within the outer two-thirds of the GCL (Villasana et al., 2015; Ibrahim et al., 2016; Tensaouti et al., 2020). IGF1 overexpression stimulated outward migration in the injured brain, yielding a significantly greater proportion of posttrauma-born immature neurons located in the middle and outer GCL in vehicle-treated IGFtg mice than in WT mice. Alterations in positioning of posttrauma-born GCL neurons are observed even as late as 6 weeks postinjury (Littlejohn et al., 2020), suggesting that new neurons that migrate to the oGCL are not eliminated. Hyperactivation of downstream effectors of IGF1 such as Akt or mTOR/S6 causes acceleration of newborn neuron migration into the outer GCL (Duan et al., 2007; Kim et al., 2009; Zhou et al., 2013). Deletion of PTEN, disinhibiting mTOR signaling, also results in increased migration of newborn granule neurons out of the inner third of the GCL (Getz et al., 2016). Increased outward migration stimulated by Akt or mTOR overactivation can be attenuated in uninjured adult mice by rapamycin treatment (Zhou et al., 2013). Here, rapamycin treatment did not significantly reduce outward migration associated with IGF1 expression, raising the possibility that additional factors such as chemokines (Schultheiss et al., 2013) or changes in guidance-related molecules are involved.

Overactivation of mTOR signaling is posited as a mediator of pathologies associated with epilepsy such as ectopic localization of newborn neurons into the HL, aberrant mossy fiber sprouting, and seizures (Meikle et al., 2007; Pun et al., 2012). Thus, elevating brain levels of IGF1 following TBI, which we show enhances early posttraumatic mTOR signaling and results in increased hippocampal neurogenesis, raises a potential concern regarding the stimulation of ectopic localization of newborn neurons and the formation of aberrant circuits. In animal models of epilepsy, inhibition of mTOR reduces mossy fiber sprouting but does not reduce ectopic hilar neurons (Buckmaster and Lew, 2011; Guo et al., 2013; Hester et al., 2016), suggesting that mislocalization of newborn neurons to the $\mathrm{HL}$ may be regulated through other pathways. We found no significant increase in ectopic localization of posttrauma-born neurons at $10 \mathrm{dpi}$ in mice with IGF1 overexpression, and no effect of rapamycin administration. In our earlier work, we show that the small proportion of newborn neurons that mislocalize to the dentate hilus after CCI in WT mice is reduced at 6 weeks postinjury in IGFtg mice (Littlejohn et al., 2020). Nonetheless, further studies are needed to examine the functional circuitry involving newborn neurons generated in the injured brain during periods of IGF1 overexpression or administration.

In addition to increased migration within the GCL, immature neurons exhibit an abnormal morphology after TBI, with a less complex dendritic arbor due to shorter dendrites and less branching (Carlson et al., 2014; Ibrahim et al., 2016; Shahror et al., 2020). In brain-injured WT mice, rapamycin treatment appeared to further impair the dendritic development of immature hippocampal neurons, consistent with a role for mTOR in regulating dendritic arborization of developing neurons during adult neurogenesis (Jaworski et al., 2005; Kim et al., 2009; Skalecka et al., 2016). In contrast, IGF1 is essential for development of neuronal dendritic arbors (Bondy and Cheng, 2004; Skalecka et al., 2016) and has been shown to rescue dendritic arborization deficits associated with developmental disorders (de Souza et al., 2017; Chen et al., 2020). We have shown that dendritic complexity of immature hippocampal neurons is significantly enhanced by IGF1 overexpression (Carlson et al., 2014). This IGF1-mediated improvement in architecture of immature neuron dendrites was observed again in the current study, and was not eliminated by rapamycin treatment.

\section{CONCLUSION AND CONSIDERATIONS}

The function and regulation of adult neurogenesis in the injured or diseased brain is not well understood. Nonetheless, harnessing endogenous neurogenesis for the replacement of damaged or dead neurons or the repair of disrupted circuitry is an attractive therapeutic target for brain injury or neurodegenerative diseases associated with hippocampal damage and deficits in hippocampally mediated behaviors such as cognition. IGF1 supports axon and dendrite growth, and promotes neurogenesis in the aging brain as well as in conditions of injury such as stroke and TBI (Madathil and Saatman, 2015). However, concerns exist regarding stimulating neurogenesis after TBI (Neuberger et al., 2017), especially because increased neurogenesis linked to seizures may underlie aberrant connectivity (Parent et al., 1997; Jessberger et al., 2005). Because IGF1 acts to increase Akt/mTOR signaling and mTOR inhibition is effective in reducing certain hallmarks of epilepsy and posttraumatic epilepsy (Buckmaster and Lew, 2011; Guo et al., 2013; Butler et al., 2015; 
Jiang et al., 2015; Hester et al., 2016), it is important to determine whether IGF1-based therapies for TBI increase the risk for posttraumatic epilepsy.

We present initial data that argues that the neurogenic effects of IGF1 in TBI do not rely on mTOR signaling. Rather, in the presence of rapamycin, the ability of IGF1 to stimulate proliferation and/or support survival of proliferated cells in the GCL as well as increase generation of new neurons is enhanced. These converging lines of evidence suggest that, under conditions of prolonged IGF1 elevation, mTOR inhibition results in disinhibition of a negative feedback loop in the IGF1 signaling pathway, resulting in greater IGF1-mediated effects on hippocampal neurogenesis. These data raise the possibility that IGF1-based therapies could be used in combination with rapamycin to stimulate functional plasticity while reducing the likelihood for posttraumatic seizures and aberrant mossy fiber sprouting. Although our previous work suggests that IGF1 contributes to improved cognitive function (Saatman et al., 1997; Madathil et al., 2013; Carlson and Saatman, 2018; Littlejohn et al., 2020), additional studies are required to assess the long-term consequences of IGF1-stimulated neurogenesis after TBI.

\section{DATA AVAILABILITY STATEMENT}

The original contributions presented in the study are included in the article/Supplementary Material. Further inquiries can be directed to the corresponding author.

\section{ETHICS STATEMENT}

The animal study was reviewed and approved by the University of Kentucky IACUC.

\section{REFERENCES}

Aberg, M. A., Aberg, N. D., Hedbacker, H., Oscarsson, J., and Eriksson, P. S. (2000). Peripheral infusion of IGF-I selectively induces neurogenesis in the adult rat hippocampus. J. Neurosci. 20, 2896-2903.

Aberg, M. A., Aberg, N. D., Palmer, T. D., Alborn, A. M., Carlsson-Skwirut, C., Bang, P., et al. (2003). IGF-I has a direct proliferative effect in adult hippocampal progenitor cells. Mol. Cell. Neurosci. 24, 23-40. doi: 10.1016/s1044-7431(03) 00082-4

Arsenijevic, Y., and Weiss, S. (1998). Insulin-like growth factor-I is a differentiation factor for postmitotic CNS stem cell-derived neuronal precursors: distinct actions from those of brain-derived neurotrophic factor. J. Neurosci. 18, 21182128.

Bekinschtein, P., Kent, B. A., Oomen, C. A., Clemenson, G. D., Gage, F. H., Saksida, L. M., et al. (2014). Brain-derived neurotrophic factor interacts with adult-born immature cells in the dentate gyrus during consolidation of overlapping memories. Hippocampus 24, 905-911. doi: 10.1002/hipo. 22304

Bharadwaj, V. N., Lifshitz, J., Adelson, P. D., Kodibagkar, V. D., and Stabenfeldt, S. E. (2016). Temporal assessment of nanoparticle accumulation after experimental brain injury: effect of particle size. Sci. Rep. 6:29988. doi: 10.1038/ srep29988

Blaiss, C. A., Yu, T. S., Zhang, G., Chen, J., Dimchev, G., Parada, L. F., et al. (2011). Temporally specified genetic ablation of neurogenesis impairs cognitive recovery after traumatic brain injury. J. Neurosci. 31, 4906-4916. doi: 10.1523/ JNEUROSCI.5265-10.2011

\section{AUTHOR CONTRIBUTIONS}

EL and KS contributed to the conception, study design, and manuscript drafting, performed data interpretation and statistical analysis. EL, AD, HW, RC, BJ, and KS performed the manuscript revisions. EL, AD, HW, RC, and JJ performed the experiments. $\mathrm{EL}, \mathrm{AD}, \mathrm{BJ}, \mathrm{RC}$, and $\mathrm{KS}$ generated the figures. EL was the first author of this work. All authors read and approved the final manuscript.

\section{FUNDING}

This work was supported by the grants from the National Institutes of Health National Institute of Neurological Disorders and Stroke (NS072302, NS092552 and NS077889) and the Kentucky Spinal Cord and Head Injury Research Trust (KSCHIRT 14-12A and 19-5A).

\section{ACKNOWLEDGMENTS}

We thank Thomas Wilkop, Associate Director of the University of Kentucky Light Microscopy Core for technical assistance regarding the Nikon A1R Confocal Microscope.

\section{SUPPLEMENTARY MATERIAL}

The Supplementary Material for this article can be found online at: https://www.frontiersin.org/articles/10.3389/fcell.2021. 663456/full\#supplementary-material

Bondy, C. A., and Cheng, C. M. (2004). Signaling by insulin-like growth factor 1 in brain. Eur. J. Pharmacol. 490, 25-31. doi: 10.1016/j.ejphar.2004.02.042

Bramlett, H. M., and Dietrich, W. D. (2015). Long-term consequences of traumatic brain injury: current status of potential mechanisms of injury and neurological outcomes. J. Neurotrauma 32, 1834-1848. doi: 10.1089/neu.2014.3352

Buckmaster, P. S., and Lew, F. H. (2011). Rapamycin suppresses mossy fiber sprouting but not seizure frequency in a mouse model of temporal lobe epilepsy. J. Neurosci. 31, 2337-2347. doi: 10.1523/JNEUROSCI.4852-10.2011

Burghardt, N. S., Park, E. H., Hen, R., and Fenton, A. A. (2012). Adult-born hippocampal neurons promote cognitive flexibility in mice. Hippocampus 22, 1795-1808. doi: 10.1002/hipo.22013

Burns, K. A., and Kuan, C. Y. (2005). Low doses of bromo- and iododeoxyuridine produce near-saturation labeling of adult proliferative populations in the dentate gyrus. Eur. J. Neurosci. 21, 803-807. doi: 10.1111/j.1460-9568.2005. 03907.x

Butler, C. R., Boychuk, J. A., and Smith, B. N. (2015). Effects of rapamycin treatment on neurogenesis and synaptic reorganization in the dentate gyrus after controlled cortical impact injury in mice. Front. Syst. Neurosci. 9:163. doi: 10.3389/fnsys.2015.00163

Bye, N., Carron, S., Han, X., Agyapomaa, D., Ng, S. Y., Yan, E., et al. (2011). Neurogenesis and glial proliferation are stimulated following diffuse traumatic brain injury in adult rats. J. Neurosci. Res. 89, 986-1000. doi: 10.1002/jnr.22635

Carloni, S., Girelli, S., Scopa, C., Buonocore, G., Longini, M., and Balduini, W. (2010). Activation of autophagy and Akt/CREB signaling play an equivalent role in the neuroprotective effect of rapamycin in neonatal hypoxia-ischemia. Autophagy 6, 366-377. doi: 10.4161/auto.6.3.11261 
Carlson, S. W., Madathil, S. K., Sama, D. M., Gao, X., Chen, J., and Saatman, K. E. (2014). Conditional overexpression of insulin-like growth factor-1 enhances hippocampal neurogenesis and restores immature neuron dendritic processes after traumatic brain injury. J. Neuropathol. Exp. Neurol. 73, 734-746. doi: 10.1097/NEN.0000000000000092

Carlson, S. W., and Saatman, K. E. (2018). Central infusion of insulin-like growth factor-1 increases hippocampal neurogenesis and improves neurobehavioral function after traumatic brain injury. J. Neurotrauma 35, 1467-1480. doi: 10 . 1089/neu.2017.5374

Carracedo, A., Ma, L., Teruya-Feldstein, J., Rojo, F., Salmena, L., Alimonti, A., et al. (2008). Inhibition of mTORC1 leads to MAPK pathway activation through a PI3K-dependent feedback loop in human cancer. J. Clin. Invest. 118, 3065-3074. doi: 10.1172/JCI34739

Chen, C. C., Jeon, S. M., Bhaskar, P. T., Nogueira, V., Sundararajan, D., Tonic, I., et al. (2010). FoxOs inhibit mTORC1 and activate Akt by inducing the expression of Sestrin 3 and rictor. Dev. Cell 18, 592-604. doi: 10.1016/j.devcel. 2010.03.008

Chen, S., Atkins, C. M., Liu, C. L., Alonso, O. F., Dietrich, W. D., and Hu, B. R. (2007). Alterations in mammalian target of rapamycin signaling pathways after traumatic brain injury. J. Cereb. Blood Flow Metab. 27, 939-949. doi: 10.1038/sj. jcbfm.9600393

Chen, S. T., Lai, W. J., Zhang, W. J., Chen, Q. P., Zhou, L. B., So, K. F., et al. (2020). Insulin-like growth factor 1 partially rescues early developmental defects caused by SHANK2 knockdown in human neurons. Neural Regen. Res. 15, 2335-2343. doi: $10.4103 / 1673-5374.285002$

Dash, P. K., Mach, S. A., and Moore, A. N. (2001). Enhanced neurogenesis in the rodent hippocampus following traumatic brain injury. J. Neurosci. Res. 63, 313-319.

de Souza, J. S., Carromeu, C., Torres, L. B., Araujo, B. H., Cugola, F. R., Maciel, R. M., et al. (2017). IGF1 neuronal response in the absence of MECP2 is dependent on TRalpha 3. Hum. Mol. Genet. 26, 270-281. doi: 10.1093/hmg/ ddw384

Dempsey, R. J., Sailor, K. A., Bowen, K. K., Tureyen, K., and Vemuganti, R. (2003). Stroke-induced progenitor cell proliferation in adult spontaneously hypertensive rat brain: effect of exogenous IGF-1 and GDNF. J. Neurochem. 87, 586-597. doi: 10.1046/j.1471-4159.2003.02022.x

Ding, K., Wang, H., Wu, Y., Zhang, L., Xu, J., Li, T., et al. (2015). Rapamycin protects against apoptotic neuronal death and improves neurologic function after traumatic brain injury in mice via modulation of the mTOR-p53-Bax axis. J. Surg. Res. 194, 239-247. doi: 10.1016/j.jss.2014.09.026

Duan, X., Chang, J. H., Ge, S., Faulkner, R. L., Kim, J. Y., Kitabatake, Y., et al. (2007). Disrupted-In-Schizophrenia 1 regulates integration of newly generated neurons in the adult brain. Cell 130, 1146-1158. doi: 10.1016/j.cell.2007.07.010

Duan, X., Kang, E., Liu, C. Y., Ming, G. L., and Song, H. (2008). Development of neural stem cell in the adult brain. Curr. Opin. Neurobiol. 18, 108-115. doi: 10.1016/j.conb.2008.04.001

Dunn-Meynell, A. A., and Levin, B. E. (1997). Histological markers of neuronal, axonal and astrocytic changes after lateral rigid impact traumatic brain injury. Brain Res. 761, 25-41. doi: 10.1016/s0006-8993(97)00210-2

Encinas, J. M., and Enikolopov, G. (2008). Identifying and quantitating neural stem and progenitor cells in the adult brain. Methods Cell Biol. 85, 243-272. doi: 10.1016/S0091-679X(08)85011-X

Erlich, S., Alexandrovich, A., Shohami, E., and Pinkas-Kramarski, R. (2007). Rapamycin is a neuroprotective treatment for traumatic brain injury. Neurobiol. Dis. 26, 86-93. doi: 10.1016/j.nbd.2006.12.003

Gao, X., and Chen, J. (2013). Moderate traumatic brain injury promotes neural precursor proliferation without increasing neurogenesis in the adult hippocampus. Exp. Neurol. 239, 38-48. doi: 10.1016/j.expneurol.2012.09.012

Gao, X., Enikolopov, G., and Chen, J. (2009). Moderate traumatic brain injury promotes proliferation of quiescent neural progenitors in the adult hippocampus. Exp. Neurol. 219, 516-523. doi: 10.1016/j.expneurol.2009.07.007

Getz, S. A., DeSpenza, T. Jr., Li, M., and Luikart, B. W. (2016). Rapamycin prevents, but does not reverse, aberrant migration in Pten knockout neurons. Neurobiol. Dis. 93, 12-20. doi: 10.1016/j.nbd.2016.03.010

Gibb, S. L., Zhao, Y., Potter, D., Hylin, M. J., Bruhn, R., Baimukanova, G., et al. (2015). TIMP3 attenuates the loss of neural stem cells, mature neurons and neurocognitive dysfunction in traumatic brain injury. Stem Cells 33, 3530-3544. doi: $10.1002 /$ stem. 2189
Goldshmit, Y., Kanner, S., Zacs, M., Frisca, F., Pinto, A. R., Currie, P. D., et al. (2015). Rapamycin increases neuronal survival, reduces inflammation and astrocyte proliferation after spinal cord injury. Mol. Cell. Neurosci. 68, 82-91. doi: 10.1016/j.mcn.2015.04.006

Guo, D., Zeng, L., Brody, D. L., and Wong, M. (2013). Rapamycin attenuates the development of posttraumatic epilepsy in a mouse model of traumatic brain injury. PLoS One 8:e64078. doi: 10.1371/journal.pone.0064078

Han, J., Wang, B., Xiao, Z., Gao, Y., Zhao, Y., Zhang, J., et al. (2008). Mammalian target of rapamycin (mTOR) is involved in the neuronal differentiation of neural progenitors induced by insulin. Mol. Cell. Neurosci. 39, 118-124. doi: 10.1016/j.mcn.2008.06.003

Harrington, L. S., Findlay, G. M., and Lamb, R. F. (2005). Restraining PI3K: mTOR signalling goes back to the membrane. Trends Biochem. Sci. 30, 35-42. doi: 10.1016/j.tibs.2004.11.003

Hartman, N. W., Lin, T. V., Zhang, L., Paquelet, G. E., Feliciano, D. M., and Bordey, A. (2013). mTORC1 targets the translational repressor 4E-BP2, but not S6 kinase 1/2, to regulate neural stem cell self-renewal in vivo. Cell Rep. 5, 433-444. doi: 10.1016/j.celrep.2013.09.017

Haruta, T., Uno, T., Kawahara, J., Takano, A., Egawa, K., Sharma, P. M., et al. (2000). A rapamycin-sensitive pathway down-regulates insulin signaling via phosphorylation and proteasomal degradation of insulin receptor substrate-1. Mol. Endocrinol. 14, 783-794. doi: 10.1210/mend.14.6.0446

He, M., Shi, X., Yang, M., Yang, T., Li, T., and Chen, J. (2019). Mesenchymal stem cells-derived IL-6 activates AMPK/mTOR signaling to inhibit the proliferation of reactive astrocytes induced by hypoxic-ischemic brain damage. Exp. Neurol. 311, 15-32. doi: 10.1016/j.expneurol.2018.09.006

Hester, M. S., Hosford, B. E., Santos, V. R., Singh, S. P., Rolle, I. J., LaSarge, C. L., et al. (2016). Impact of rapamycin on status epilepticus induced hippocampal pathology and weight gain. Exp. Neurol. 280, 1-12. doi: 10.1016/j.expneurol. 2016.03.015

Hurtado-Chong, A., Yusta-Boyo, M. J., Vergano-Vera, E., Bulfone, A., de Pablo, F., and Vicario-Abejon, C. (2009). IGF-I promotes neuronal migration and positioning in the olfactory bulb and the exit of neuroblasts from the subventricular zone. Eur. J. Neurosci. 30, 742-755. doi: 10.1111/j.1460-9568. 2009.06870.x

Ibrahim, S., Hu, W., Wang, X., Gao, X., He, C., and Chen, J. (2016). Traumatic brain injury causes aberrant migration of adult-born neurons in the hippocampus. Sci. Rep. 6:21793. doi: 10.1038/srep21793

Jaworski, J., Spangler, S., Seeburg, D. P., Hoogenraad, C. C., and Sheng, M. (2005). Control of dendritic arborization by the phosphoinositide-3'-kinaseAkt-mammalian target of rapamycin pathway. J. Neurosci. 25, 11300-11312. doi: 10.1523/JNEUROSCI.2270-05.2005

Jessberger, S., Romer, B., Babu, H., and Kempermann, G. (2005). Seizures induce proliferation and dispersion of doublecortin-positive hippocampal progenitor cells. Exp. Neurol. 196, 342-351. doi: 10.1016/j.expneurol.2005.08.010

Jiang, G., Wang, W., Cao, Q., Gu, J., Mi, X., Wang, K., et al. (2015). Insulin growth factor-1 (IGF-1) enhances hippocampal excitatory and seizure activity through IGF-1 receptor-mediated mechanisms in the epileptic brain. Clin. Sci. 129, 1047-1060. doi: 10.1042/CS20150312

Kempermann, G., Gast, D., Kronenberg, G., Yamaguchi, M., and Gage, F. H. (2003). Early determination and long-term persistence of adult-generated new neurons in the hippocampus of mice. Development 130, 391-399.

Kempermann, G., Song, H., and Gage, F. H. (2015). Neurogenesis in the adult hippocampus. Cold Spring Harb. Perspect. Biol. 7:a018812. doi: 10.1101/ cshperspect.a018812

Kernie, S. G., Erwin, T. M., and Parada, L. F. (2001). Brain remodeling due to neuronal and astrocytic proliferation after controlled cortical injury in mice. J. Neurosci. Res. 66, 317-326. doi: 10.1002/jnr.10013

Kernie, S. G., and Parent, J. M. (2010). Forebrain neurogenesis after focal Ischemic and traumatic brain injury. Neurobiol. Dis. 37, 267-274. doi: 10.1016/j.nbd. 2009.11.002

Kim, J. Y., Duan, X., Liu, C. Y., Jang, M. H., Guo, J. U., Pow-anpongkul, N., et al. (2009). DISC1 regulates new neuron development in the adult brain via modulation of AKT-mTOR signaling through KIAA1212. Neuron 63, 761-773. doi: 10.1016/j.neuron.2009.08.008

Kuhn, H. G., Dickinson-Anson, H., and Gage, F. H. (1996). Neurogenesis in the dentate gyrus of the adult rat: age-related decrease of neuronal progenitor proliferation. J. Neurosci. 16, 2027-2033. 
Lipton, J. O., and Sahin, M. (2014). The neurology of mTOR. Neuron 84, 275-291. doi: 10.1016/j.neuron.2014.09.034

Littlejohn, E. L., Scott, D., and Saatman, K. E. (2020). Insulin-like growth factor-1 overexpression increases long-term survival of posttrauma-born hippocampal neurons while inhibiting ectopic migration following traumatic brain injury. Acta Neuropathol. Commun. 8:46. doi: 10.1186/s40478-020-00925-6

Madathil, S. K., Carlson, S. W., Brelsfoard, J. M., Ye, P., D’Ercole, A. J., and Saatman, K. E. (2013). Astrocyte-specific overexpression of insulin-like growth factor-1 protects hippocampal neurons and reduces behavioral deficits following traumatic brain injury in mice. PLoS One 8:e67204. doi: 10.1371/ journal.pone.0067204

Madathil, S. K., and Saatman, K. E. (2015). "IGF-1/IGF-R signaling in traumatic brain injury: impact on cell survival, neurogenesis, and behavioral outcome," in Brain Neurotrauma: Molecular, Neuropsychological, and Rehabilitation Aspects, ed. F. H. Kobeissy (Boca Raton, FL: Wiely).

Mathews, E. A., Morgenstern, N. A., Piatti, V. C., Zhao, C., Jessberger, S., Schinder, A. F., et al. (2010). A distinctive layering pattern of mouse dentate granule cells is generated by developmental and adult neurogenesis. J. Comp. Neurol. 518, 4479-4490. doi: 10.1002/cne.22489

McCurdy, R. D., Feron, F., McGrath, J. J., and Mackay-Sim, A. (2005). Regulation of adult olfactory neurogenesis by insulin-like growth factor-I. Eur. J. Neurosci. 22, 1581-1588. doi: 10.1111/j.1460-9568.2005.04355.x

McGinn, M. J., and Povlishock, J. T. (2015). Cellular and molecular mechanisms of injury and spontaneous recovery. Handb. Clin. Neurol. 127, 67-87. doi: 10.1016/B978-0-444-52892-6.00005-2

Meikle, L., Talos, D. M., Onda, H., Pollizzi, K., Rotenberg, A., Sahin, M., et al. (2007). A mouse model of tuberous sclerosis: neuronal loss of Tscl causes dysplastic and ectopic neurons, reduced myelination, seizure activity, and limited survival. J. Neurosci. 27, 5546-5558. doi: 10.1523/JNEUROSCI.554006.2007

Millan, O., Wieland, E., Marquet, P., and Brunet, M. (2019). Pharmacodynamic Monitoring of mTOR Inhibitors. Ther. Drug Monit. 41, 160-167. doi: 10.1097/ FTD.0000000000000616

Myer, D. J., Gurkoff, G. G., Lee, S. M., Hovda, D. A., and Sofroniew, M. V. (2006). Essential protective roles of reactive astrocytes in traumatic brain injury. Brain 129(Pt 10), 2761-2772. doi: 10.1093/brain/awl165

Neuberger, E. J., Swietek, B., Corrubia, L., Prasanna, A., and Santhakumar, V. (2017). Enhanced dentate neurogenesis after brain injury undermines longterm neurogenic potential and promotes seizure susceptibility. Stem Cell Rep. 9, 972-984. doi: 10.1016/j.stemcr.2017.07.015

Nieto-Estevez, V., Defterali, C., and Vicario-Abejon, C. (2016). IGF-I: a key growth factor that regulates neurogenesis and synaptogenesis from embryonic to adult stages of the brain. Front. Neurosci. 10:52. doi: 10.3389/fnins.2016.00052

Nikolaeva, I., Crowell, B., Valenziano, J., Meaney, D., and D’Arcangelo, G. (2016). Beneficial effects of early mTORC1 inhibition after traumatic brain injury. J. Neurotrauma 33, 183-193. doi: 10.1089/neu.2015.3899

O'Kusky, J., and Ye, P. (2012). Neurodevelopmental effects of insulin-like growth factor signaling. Front. Neuroendocrinol. 33, 230-251. doi: 10.1016/j.yfrne.2012. 06.002

O’Reilly, K. E., Rojo, F., She, Q. B., Solit, D., Mills, G. B., Smith, D., et al. (2006). mTOR inhibition induces upstream receptor tyrosine kinase signaling and activates Akt. Cancer Res. 66, 1500-1508. doi: 10.1158/0008-5472.CAN-052925

Orhan, N., Ugur Yilmaz, C., Ekizoglu, O., Ahishali, B., Kucuk, M., Arican, N., et al. (2016). Effects of beta-hydroxybutyrate on brain vascular permeability in rats with traumatic brain injury. Brain Res. 1631, 113-126. doi: 10.1016/j.brainres. 2015.11.038

Otaegi, G., Yusta-Boyo, M. J., Vergano-Vera, E., Mendez-Gomez, H. R., Carrera, A. C., Abad, J. L., et al. (2006). Modulation of the PI 3-kinase-Akt signalling pathway by IGF-I and PTEN regulates the differentiation of neural stem/precursor cells. J. Cell Sci. 119(Pt 13), 2739-2748. doi: 10.1242/jcs.03012

Paliouras, G. N., Hamilton, L. K., Aumont, A., Joppe, S. E., Barnabe-Heider, F., and Fernandes, K. J. (2012). Mammalian target of rapamycin signaling is a key regulator of the transit-amplifying progenitor pool in the adult and aging forebrain. J. Neurosci. 32, 15012-15026. doi: 10.1523/JNEUROSCI.2248-12. 2012

Parent, J. M., Yu, T. W., Leibowitz, R. T., Geschwind, D. H., Sloviter, R. S., and Lowenstein, D. H. (1997). Dentate granule cell neurogenesis is increased by seizures and contributes to aberrant network reorganization in the adult rat hippocampus. J. Neurosci. 17, 3727-3738.

Park, J., Zhang, J., Qiu, J., Zhu, X., Degterev, A., Lo, E. H., et al. (2012). Combination therapy targeting Akt and mammalian target of rapamycin improves functional outcome after controlled cortical impact in mice. J. Cereb. Blood Flow Metab. 32, 330-340. doi: 10.1038/jcbfm.2011.131

Peters, A. J., Villasana, L. E., and Schnell, E. (2018). Ketamine alters hippocampal cell proliferation and improves learning in mice after traumatic brain injury. Anesthesiology 129, 278-295. doi: 10.1097/ALN.0000000000002197

Plumpe, T., Ehninger, D., Steiner, B., Klempin, F., Jessberger, S., Brandt, M., et al. (2006). Variability of doublecortin-associated dendrite maturation in adult hippocampal neurogenesis is independent of the regulation of precursor cell proliferation. BMC Neurosci. 7:77. doi: 10.1186/1471-2202-7-77

Pun, R. Y., Rolle, I. J., Lasarge, C. L., Hosford, B. E., Rosen, J. M., Uhl, J. D., et al. (2012). Excessive activation of mTOR in postnatally generated granule cells is sufficient to cause epilepsy. Neuron 75, 1022-1034. doi: 10.1016/j.neuron.2012. 08.002

Robinson, C., Apgar, C., and Shapiro, L. A. (2016). Astrocyte hypertrophy contributes to aberrant neurogenesis after traumatic brain injury. Neural Plast. 2016:1347987. doi: 10.1155/2016/1347987

Rola, R., Mizumatsu, S., Otsuka, S., Morhardt, D. R., Noble-Haeusslein, L. J., Fishman, K., et al. (2006). Alterations in hippocampal neurogenesis following traumatic brain injury in mice. Exp. Neurol. 202, 189-199. doi: 10.1016/j. expneurol.2006.05.034

Rozas, N. S., Redell, J. B., Hill, J. L., McKenna, J. III, Moore, A. N., Gambello, M. J., et al. (2015). Genetic activation of mTORC1 signaling worsens neurocognitive outcome after traumatic brain injury. J. Neurotrauma 32, 149-158. doi: 10.1089/ neu.2014.3469

Saatman, K. E., Contreras, P. C., Smith, D. H., Raghupathi, R., McDermott, K. L., Fernandez, S. C., et al. (1997). Insulin-like growth factor-1 (IGF-1) improves both neurological motor and cognitive outcome following experimental brain injury. Exp. Neurol. 147, 418-427. doi: 10.1006/exnr.1997.6629

Saatman, K. E., Feeko, K. J., Pape, R. L., and Raghupathi, R. (2006). Differential behavioral and histopathological responses to graded cortical impact injury in mice. J. Neurotrauma 23, 1241-1253. doi: 10.1089/neu.2006.23.1241

Sahay, A., Scobie, K. N., Hill, A. S., O'Carroll, C. M., Kheirbek, M. A., Burghardt, N. S., et al. (2011). Increasing adult hippocampal neurogenesis is sufficient to improve pattern separation. Nature 472, 466-470. doi: 10.1038/nature0 9817

Schultheiss, C., Abe, P., Hoffmann, F., Mueller, W., Kreuder, A. E., Schutz, D., et al. (2013). CXCR4 prevents dispersion of granule neuron precursors in the adult dentate gyrus. Hippocampus 23, 1345-1358. doi: 10.1002/hipo.22180

Shahror, R. A., Linares, G. R., Wang, Y., Hsueh, S. C., Wu, C. C., Chuang, D. M., et al. (2020). Transplantation of mesenchymal stem cells overexpressing fibroblast growth factor 21 facilitates cognitive recovery and enhances neurogenesis in a mouse model of traumatic brain injury. J. Neurotrauma 37 , 14-26. doi: 10.1089/neu.2019.6422

Shapiro, L. A. (2017). Altered hippocampal neurogenesis during the first 7 days after a fluid percussion traumatic brain injury. Cell Transplant. 26, 1314-1318. doi: 10.1177/0963689717714099

Shi, J., Miles, D. K., Orr, B. A., Massa, S. M., and Kernie, S. G. (2007). Injuryinduced neurogenesis in Bax-deficient mice: evidence for regulation by voltagegated potassium channels. Eur. J. Neurosci. 25, 3499-3512. doi: 10.1111/j.14609568.2007.05624.x

Skalecka, A., Liszewska, E., Bilinski, R., Gkogkas, C., Khoutorsky, A., Malik, A. R., et al. (2016). mTOR kinase is needed for the development and stabilization of dendritic arbors in newly born olfactory bulb neurons. Dev. Neurobiol. 76, 1308-1327. doi: 10.1002/dneu.22392

Song, Q., Xie, D., Pan, S., and Xu, W. (2015). Rapamycin protects neurons from brain contusioninduced inflammatory reaction via modulation of microglial activation. Mol. Med. Rep. 12, 7203-7210. doi: 10.3892/mmr.2015.4407

Sun, D., Colello, R. J., Daugherty, W. P., Kwon, T. H., McGinn, M. J., Harvey, H. B., et al. (2005). Cell proliferation and neuronal differentiation in the dentate gyrus in juvenile and adult rats following traumatic brain injury. J. Neurotrauma 22, 95-105. doi: 10.1089/neu.2005.22.95

Taupin, P. (2007). BrdU immunohistochemistry for studying adult neurogenesis: paradigms, pitfalls, limitations, and validation. Brain Res. Rev. 53, 198-214. doi: 10.1016/j.brainresrev.2006.08.002 
Taylor, C. A., Bell, J. M., Breiding, M. J., and Xu, L. (2017). Traumatic Brain Injury-Related Emergency Department Visits, Hospitalizations, and Deaths United States, 2007 and 2013. MMWR Surveill. Summ. 66, 1-16. doi: 10.15585/ mmwr.ss6609a1

Tensaouti, Y., Yu, T. S., and Kernie, S. G. (2020). Apolipoprotein E regulates the maturation of injury-induced adult-born hippocampal neurons following traumatic brain injury. PLoS One 15:e229240. doi: 10.1371/journal.pone. 0229240

Villasana, L. E., Kim, K. N., Westbrook, G. L., and Schnell, E. (2015). Functional integration of adult-born hippocampal neurons after traumatic brain injury(1,2,3). eNeuro 2:ENEURO.0056-15.2015. doi: 10.1523/ENEURO. 0056-15.2015

Wahane, S. D., Hellbach, N., Prentzell, M. T., Weise, S. C., Vezzali, R., Kreutz, C., et al. (2014). PI3K-p110-alpha-subtype signalling mediates survival, proliferation and neurogenesis of cortical progenitor cells via activation of mTORC2. J. Neurochem. 130, 255-267. doi: 10.1111/jnc. 12718

Wang, X., Seekaew, P., Gao, X., and Chen, J. (2016). Traumatic brain injury stimulates neural stem cell proliferation via mammalian target of rapamycin signaling pathway activation. eNeuro 3:ENEURO.0162-16.2016. doi: 10.1523/ ENEURO.0162-16.2016

Ye, P., Popken, G. J., Kemper, A., McCarthy, K., Popko, B., and D'Ercole, A. J. (2004). Astrocyte-specific overexpression of insulin-like growth factorI promotes brain overgrowth and glial fibrillary acidic protein expression. J. Neurosci. Res. 78, 472-484. doi: 10.1002/jnr.20288

Yu, M., Wang, M., Yang, D., Wei, X., and Li, W. (2019). Dynamics of blood brain barrier permeability and tissue microstructure following controlled cortical impact injury in rat: a dynamic contrast-enhanced magnetic resonance imaging and diffusion kurtosis imaging study. Magn. Reson. Imaging 62, 1-9.

Yu, T. S., Zhang, G., Liebl, D. J., and Kernie, S. G. (2008). Traumatic brain injury-induced hippocampal neurogenesis requires activation of early nestin-expressing progenitors. J. Neurosci. 28, 12901-12912. doi: 10.1523/ JNEUROSCI.4629-08.2008

Zhang, X., Zhang, L., Cheng, X., Guo, Y., Sun, X., Chen, G., et al. (2014). IGF-1 promotes Brn-4 expression and neuronal differentiation of neural stem cells via the PI3K/Akt pathway. PLoS One 9:e113801. doi: 10.1371/journal.pone. 0113801

Zhou, M., Li, W., Huang, S., Song, J., Kim, J. Y., Tian, X., et al. (2013). mTOR Inhibition ameliorates cognitive and affective deficits caused by Disc1 knockdown in adult-born dentate granule neurons. Neuron 77, 647-654. doi: 10.1016/j.neuron.2012.12.033

Zhu, G., Chow, L. M., Bayazitov, I. T., Tong, Y., Gilbertson, R. J., Zakharenko, S. S., et al. (2012). Pten deletion causes mTorc1-dependent ectopic neuroblast differentiation without causing uniform migration defects. Development 139 , 3422-3431. doi: 10.1242/dev.083154

Zhu, X., Park, J., Golinski, J., Qiu, J., Khuman, J., Lee, C. C., et al. (2014). Role of Akt and mammalian target of rapamycin in functional outcome after concussive brain injury in mice. J. Cereb. Blood Flow Metab. 34, 1531-1539. doi: 10.1038/ jcbfm.2014.113

Conflict of Interest: The authors declare that the research was conducted in the absence of any commercial or financial relationships that could be construed as a potential conflict of interest.

Copyright (C) 2021 Littlejohn, DeSana, Williams, Chapman, Joseph, Juras and Saatman. This is an open-access article distributed under the terms of the Creative Commons Attribution License (CC BY). The use, distribution or reproduction in other forums is permitted, provided the original author(s) and the copyright owner(s) are credited and that the original publication in this journal is cited, in accordance with accepted academic practice. No use, distribution or reproduction is permitted which does not comply with these terms. 